\title{
Outflows and accretion in a star-disc system with stellar magnetosphere and disc dynamo
}

\author{
B. von Rekowski ${ }^{1}$ and A. Brandenburg ${ }^{2}$ \\ 1 Department of Astronomy \& Space Physics, Uppsala University, Box 515, 75120 Uppsala, Sweden \\ 2 NORDITA, Blegdamsvej 17, 2100 Copenhagen Ø, Denmark
}

Received 10 July 2003 / Accepted 3 March 2004

\begin{abstract}
The interaction between a protostellar magnetosphere and a surrounding dynamo-active accretion disc is investigated using an axisymmetric mean-field model. In all models investigated, the dynamo-generated magnetic field in the disc arranges itself such that in the corona, the field threading the disc is anti-aligned with the central dipole so that no X-point forms. When the magnetospheric field is strong enough (stellar surface field strength around $2 \mathrm{kG}$ or larger), accretion happens in a recurrent fashion with periods of around 15 to 30 days, which is somewhat longer than the stellar rotation period of around 10 days. In the case of a stellar surface field strength of at least a few $100 \mathrm{G}$, the star is being spun up by the magnetic torque exerted on the star. The stellar accretion rates are always reduced by the presence of a magnetosphere which tends to divert a much larger fraction of the disc material into the wind. Both, a pressure-driven stellar wind and a disc wind form. In all our models with disc dynamo, the disc wind is structured and driven by magneto-centrifugal as well as pressure forces.
\end{abstract}

Key words. ISM: jets and outflows - accretion, accretion disks - magnetic fields - magnetohydrodynamics (MHD)

\section{Introduction}

The interaction of a stellar magnetic field with a circumstellar accretion disc was originally studied in connection with accretion discs around neutron stars (Ghosh et al. 1977; Ghosh \& Lamb 1979a,b), but it was later also applied to protostellar magnetospheres (Königl 1991; Cameron \& Campbell 1993; Shu et al. 1994). Most of the work is based on the assumption that the field in the disc is constantly being dragged into the inner parts of the disc from large radii. The idea behind this is that a magnetized molecular cloud collapses, in which case the field in the central star and that in the disc are aligned (Shu et al. 1994). This was studied numerically by Hirose et al. (1997) and Miller \& Stone (1997). In the configurations they considered, there is an X-point in the equatorial plane (see left hand panel of Fig. 1), which can lead to a strong funnel flow.

Another alternative has been explored by Lovelace et al. (1995) where the magnetic field of the star has been flipped and is now anti-parallel with the field in the disc, so that the field in the equatorial plane points in the same direction and has no $\mathrm{X}$-point. However, a current sheet develops above and below the disc plane (see right hand panel of Fig. 1). Numerical simulations of such a field configuration by Hayashi et al. (1996) confirm the idea by Lovelace et al. (1995) that closed magnetic loops connecting the star and the disc are twisted by differential rotation between the star and the disc, and then

Send offprint requests to: B. von Rekowski,

e-mail: Brigitta.vonRekowski@astro.uu.se inflate to form open stellar and disc field lines (see also Bardou 1999; Agapitou \& Papaloizou 2000). Goodson et al. (1997, 1999) and Goodson \& Winglee (1999) show that for sufficiently low resistivity, an accretion process develops that is unsteady and proceeds in an oscillatory fashion. The inflating magnetosphere expands to larger radii where matter can be loaded onto the field lines and be ejected as stellar and disc winds. Reconnection of magnetic field lines allows matter to flow along them and accrete onto the protostar, in the form of a funnel flow (see also Romanova et al. 2002). Consequently, their stellar jets show episodic behaviour; see also Matt et al. (2002).

The gas in the disc is turbulent and hence capable of converting part of the kinetic energy into magnetic energy by dynamo action. Furthermore, the differential rotation of the disc can allow for large scale magnetic fields, possibly with spatiotemporal order (similar to the solar 11 year cycle; see, e.g., Parker 1979). Such a dynamo may have operated in the solar nebula and in circumstellar or protoplanetary discs (e.g., Reyes-Ruiz \& Stepinski 1995). However, not much is known about the mutual interaction between a stellar magnetic field and a dynamo-generated disc field. In particular, we need to understand the effect of the magnetic field generated in the disc, and we also want to know how this is being affected by the presence of the central dipole field. Following a recent attempt to model outflows from cool, dynamo-active accretion discs (von Rekowski et al. 2003; hereafter referred to as Paper I), we present in this paper a study of the interaction between the 

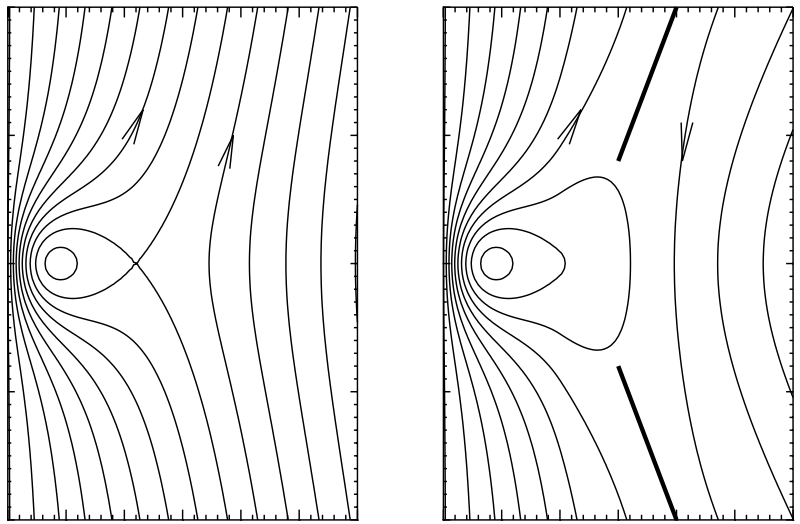

Fig. 1. Sketch showing the formation of an X-point when the disc field is aligned with the dipole (on the left) and the formation of current sheets with no X-point if they are anti-aligned (on the right). The two current sheets are shown as thick lines. In the present paper, the second of the two configurations emerges in all our models, i.e. with current sheets and no X-point.

dynamo-generated disc magnetic field and the field of the central star. In our model, both the star and the disc have a wind, resulting in a structured outflow that is driven by a combination of different processes including pressure-driving as well as magneto-centrifugal acceleration.

\section{The magnetospheric model}

A detailed description of the model without magnetosphere can be found in Sect. 2 of Paper I. We begin the description of the present model by reviewing the basic setup of the model in Paper I. The implementation of the stellar magnetosphere is described in Sect. 2.4.

\subsection{Basic equations}

We solve the set of axisymmetric MHD equations in cylindrical polar coordinates $(\varpi, \varphi, z)$, consisting of the continuity equation,

$$
\frac{\partial \varrho}{\partial t}+\nabla \cdot(\varrho \boldsymbol{u})=q_{\varrho}^{\mathrm{disc}}+q_{\varrho}^{\mathrm{star}},
$$

the Navier-Stokes equation,

$$
\frac{\mathrm{D} \boldsymbol{u}}{\mathrm{D} t}=-\frac{1}{\varrho} \boldsymbol{\nabla} p-\nabla \Phi+\frac{1}{\varrho}\left[\boldsymbol{F}+\left(\boldsymbol{u}_{\mathrm{K}}-\boldsymbol{u}\right) q_{\varrho}^{\mathrm{disc}}\right],
$$

and the mean field induction equation,

$$
\frac{\partial \boldsymbol{A}}{\partial t}=\boldsymbol{u} \times \boldsymbol{B}+\alpha \boldsymbol{B}-\eta \mu_{0} \boldsymbol{J} .
$$

Here, $\boldsymbol{u}$ is the velocity field, $\varrho$ is the gas density, $p$ is the gas pressure, $\Phi$ is the gravitational potential, $t$ is time, $\mathrm{D} / \mathrm{D} t=$ $\partial / \partial t+\boldsymbol{u} \cdot \boldsymbol{\nabla}$ is the advective derivative, $\boldsymbol{F}=\boldsymbol{J} \times \boldsymbol{B}+\boldsymbol{\nabla} \cdot \boldsymbol{\tau}$ is the sum of the Lorentz and viscous forces, $\boldsymbol{J}=\boldsymbol{\nabla} \times \boldsymbol{B} / \mu_{0}$ is the current density due to the mean magnetic field $\boldsymbol{B}, \mu_{0}$ is the magnetic permeability, and $\tau$ is the (isotropic) viscous stress tensor. In the disc, we assume a turbulent (Shakura-Sunyaev) viscosity, $v_{\mathrm{t}}=\alpha_{\mathrm{SS}} c_{\mathrm{S}} z_{0}$, where $\alpha_{\mathrm{SS}}$ is the Shakura-Sunyaev coefficient (less than unity), $c_{\mathrm{s}}=(\gamma p / \varrho)^{1 / 2}$ is the sound speed, $\gamma=c_{\mathrm{p}} / c_{\mathrm{v}}$ is the ratio of the specific heat at constant pressure, $c_{\mathrm{p}}$, and the specific heat at constant volume, $c_{\mathrm{v}}$, and $z_{0}$ is the disc half-thickness.

As described in Paper I, in order to maintain a statistically steady accretion disc, we need to replenish the mass that is accreted through the disc and onto the star. We therefore include a mass source, $q_{\varrho}^{\text {disc }}$, that is restricted to the disc and selfregulatory, i.e. it turns on once the local density in the disc drops below the initial density distribution, $\varrho_{0}(\boldsymbol{r})$, of the hydrostatic equilibrium. This means that matter is injected into the disc only wherever and whenever $\varrho<\varrho_{0}$, and the strength of the mass source is proportional to the gas density deficit. Therefore, we do not prescribe the distribution and magnitude of the mass source beforehand, but the system adjusts itself. We also allow for a self-regulatory mass $\operatorname{sink}, q_{\varrho}^{\text {star }}$, at the position of the central object (protostar) to model accretion onto the central star without changing the stellar radius. The mass sink is modelled in a way analogous to the mass source. In the models with magnetosphere, however, a mass sink in the star is modelled by setting density, velocity and magnetic field in the star to their initial values at each timestep. This is necessary in order to anchor the magnetosphere in the star (cf. Sect. 2.4). The mass source appears also in the Navier-Stokes equation, unless matter is injected with the ambient velocity of the gas. We always inject matter with Keplerian speed, $\boldsymbol{u}_{\mathrm{K}}$. This leads to an extra term in the Navier-Stokes equation, $\left(\boldsymbol{u}_{\mathrm{K}}-\boldsymbol{u}\right) q_{\varrho}^{\mathrm{disc}}$, which vanishes only if the gas rotates already with Keplerian speed or when no mass is injected $\left(q_{\varrho}^{\text {disc }}=0\right)$.

A physically realistic accretion disc is much more strongly stratified than what can be represented in the simulations. We expect most of the actual accretion to occur in the innermost parts of the disc. These inner parts are also much cooler and therefore they should be spinning at almost exactly Keplerian speed, i.e. usually faster than the outer parts of the disc. This is the reason why we choose to inject new matter at Keplerian speed. One additional reason why we do not inject matter with the ambient gas velocity is that we want to prevent a runaway effect (cf. Sect. 2.1 in Paper I). Such a runaway could result in a loss of the initial angular momentum of the disc, which would never be replenished, so that the entire disc would eventually lose its angular momentum and all matter would be accreted. However, in some cases we have compared simulations with and without Keplerian injection and found the difference to be small.

Furthermore, mass replenishment in our disc is necessary, because we model an accretion disc that is truncated at about 0.19 AU (cf. Sect. 2.3), i.e. we model the inner part of a realistic (protostellar) accretion disc. The total run time of our simulations is between $\sim 150$ days (Model S) and $\sim 1900$ days (Model M1), corresponding to about 33 to 422 Keplerian orbital periods of the inner disc edge (cf. Sects. 2.3 and 3). This is shorter than the life time of the inner part of a protostellar accretion disc. On the other hand, a typical advection time in our models is $\left(\varpi_{\text {out }}-\varpi_{\text {in }}\right) / u_{\varpi} \approx(1.9-0.6) \times 0.1 \mathrm{AU} /(0.15 \times$ $10^{2} \mathrm{~km} \mathrm{~s}^{-1}$ ) $\approx 15$ days (cf. Sects. 2.3 and 3.5), which is much shorter than the typical run time so that our disc would 
disappear if there were no mass supply. One way to model mass accretion from the outer parts of the disc to the inner parts, is to extend our disc to the radial boundary of our computational domain and to inject matter at this radial boundary. Another way - that we have chosen - is to inject matter locally in the disc wherever and whenever it is needed.

The technique of invoking self-regulatory terms that act in certain parts of the computational domain is an alternative to prescribing boundary conditions. Similar techniques have been used in modelling the Earth's magnetosphere (Janhunen \& Huuskonen 1993) and in magnetized Couette flow problems where inner and outer cylinders are present as part of a cartesian mesh (Dobler et al. 2002). In the present case, our star and accretion disc play a hybrid rôle in that they represent not only boundary conditions to the corona including the region between disc and star. In the models without magnetosphere, the star is not only a region where mass is being absorbed by the mass sink, but one can also study the dynamics of the velocity and magnetic fields in the star and the resulting effects. We do not prescribe inflow at the star's surface; instead, our model allows for both inflow and outflow at different parts of the star's surface. In the models with magnetosphere, the star remaining within the mesh allows one to study the effects of a spinning dipole. The disc surface is not an outflow boundary condition, i.e. a region where mass is being injected into the corona, but the outflow develops itself. Resolving the disc makes it possible to include a disc dynamo which is the innovative ingredient in the models of Paper I and the present paper. Our modelling of the disc dynamo will be explained in the following section.

We assume that the magnetic field in the disc be generated by a standard $\alpha^{2} \Omega$ dynamo (e.g., Krause \& Rädler 1980), where $\alpha$ is the mean-field $\alpha$ effect and $\Omega$ is the angular velocity of the plasma. This implies an extra electromotive force, $\alpha \boldsymbol{B}$, in the induction equation for the mean magnetic field, $\boldsymbol{B}$, that is restricted to the disc. As usual, the $\alpha$ effect is antisymmetric about the midplane with (see Paper I)

$\alpha=\alpha_{0} \frac{z}{z_{0}} \frac{\xi_{\text {disc }}(\boldsymbol{r})}{1+v_{\mathrm{A}}^{2} / c_{\mathrm{s}}^{2}}$,

where $v_{\mathrm{A}}$ is the Alfvén speed based on the total magnetic field, $\xi_{\text {disc }}$ is a profile specifying the shape of the disc (see Sect. 2.2), and $\alpha_{0}$ is a parameter that controls the intensity of dynamo action. We choose the $\alpha$ effect to be negative in the upper half of the disc (i.e. $\alpha_{0}<0$ ), consistent with results from simulations of accretion disc turbulence driven by the magneto-rotational instability (Brandenburg et al. 1995; Ziegler \& Rüdiger 2000). The resulting magnetic field symmetry is roughly dipolar. This symmetry is seen both in threedimensional simulations of accretion disc dynamos driven by turbulence from the magneto-rotational instability and in solutions of the $\alpha \Omega$ dynamo problem with the $\alpha$ effect negative in the upper disc half, provided that the accretion disc is embedded in a conducting corona (e.g., Brandenburg et al. 1990; Brandenburg 1998; von Rekowski et al. 2000; Bardou et al. 2001). Further, we include $\alpha$ quenching which leads to the disc dynamo saturating at a level close to equipartition between magnetic and thermal energies. The (constant) magnetic diffusivity $\eta$ is finite in the whole domain, and enhanced in the disc by turbulence. We do not take into account that the diffusivity in the star might also be enhanced due to convection but assume the same value in the star as in the corona.

To ensure that $\boldsymbol{B}$ is solenoidal, we solve the induction equation in terms of the vector potential $\boldsymbol{A}$, where $\boldsymbol{B}=\boldsymbol{\nabla} \times \boldsymbol{A}$.

We impose regularity conditions on the axis $(\varpi=0)$ and outflow boundary conditions on $\varpi=\varpi_{\max }$ and $z= \pm z_{\max }$.

\subsection{A cool disc in a hot corona: The initial state}

A simple way to implement a cool, dense disc embedded in a hot, rarefied corona without modelling the detailed physics of coronal heating is to prescribe the specific entropy, $s$, such that $s$ is smaller within the disc and larger in the corona. For a perfect gas this implies $p=\mathrm{e}^{s / c_{\mathrm{v}}} \varrho^{\gamma}$ (in a dimensionless form), with the polytrope parameter $\mathrm{e}^{s / c_{\mathrm{v}}}$ being a function of position. In the model considered here, we have an intermediate value for the specific entropy within the star. We prescribe the polytrope parameter to be unity in the corona and less than unity in the disc and in the star, so we put

$\mathrm{e}^{s / c_{\mathrm{p}}}=1-\left(1-\beta_{\text {disc }}\right) \xi_{\text {disc }}-\left(1-\beta_{\text {star }}\right) \xi_{\text {star }}$,

where $\xi_{\text {disc }}$ and $\xi_{\text {star }}$ are (time-independent) profiles specifying the shapes of the disc and the star. The free parameters, $0<$ $\beta_{\text {disc }}, \beta_{\text {star }}<1$, control the entropy contrasts between disc and corona and between star and corona, respectively.

In the absence of a magnetosphere, our initial state is a hydrostatic equilibrium with no poloidal velocity, assuming an initially non-rotating hot corona that is supported by the pressure gradient. Since we model a disc that is cool, the disc is mainly centrifugally supported, and as a result it is rotating at slightly sub-Keplerian speed.

The temperature ratio between disc and corona is roughly $\beta_{\text {disc }}$. Assuming pressure equilibrium between disc and corona, and $p \propto \rho T$ for a perfect gas, the corresponding density ratio is then $\beta_{\text {disc }}^{-1}$. Thus, the entropy contrast between disc and corona chosen here $\left(\beta_{\text {disc }}=0.005\right)$, leads to density and inverse temperature ratios of 200:1 between disc and corona.

A rough estimate for the initial toroidal velocity, $u_{\varphi 0}$, in the midplane of the disc follows from the hydrostatic equilibrium as $u_{\varphi 0} \approx \sqrt{1-\beta_{\mathrm{disc}}} \boldsymbol{u}_{\mathrm{K}}$. For $\beta_{\mathrm{disc}}=0.005$, the toroidal velocity is within $0.25 \%$ of the Keplerian speed.

The initial hydrostatic solution is an unstable equilibrium because of the vertical shear between the disc, star and corona (Urpin \& Brandenburg 1998). In addition, angular momentum transfer by viscous and magnetic stresses - the latter from the disc dynamo - drives the solution immediately away from the initial state.

\subsection{Application to a protostellar star-disc system}

Since we use dimensionless variables, our model can be rescaled and can therefore be applied to a range of different astrophysical objects. Here, we consider values for our normalization parameters that are typical of a protostellar stardisc system. We scale the sound speed with a typical coronal 
sound speed of $c_{\mathrm{s} 0}=10^{2} \mathrm{~km} \mathrm{~s}^{-1}$, which corresponds to a temperature of $T_{0} \approx 4 \times 10^{5} \mathrm{~K}$, and the disc surface density with $\Sigma_{0}=1 \mathrm{~g} \mathrm{~cm}^{-2}$. Further, we assume $M_{*}=1 M_{\odot}$ (where $M_{*}$ and $M_{\odot}$ are the stellar and solar mass, respectively), a mean specific weight of $\mu=0.6$, and $\gamma=5 / 3$. This fixes the units of all quantities. The resulting velocity unit is $[\boldsymbol{u}]=c_{\mathrm{s} 0}=10^{2} \mathrm{~km} \mathrm{~s}^{-1}$, the length unit is $[\boldsymbol{r}]=$ $G M_{*} /[\boldsymbol{u}]^{2} \approx 0.1 \mathrm{AU}$, the time unit is $[t]=[\boldsymbol{r}] /[\boldsymbol{u}] \approx 1.5 \mathrm{~d}$, the unit for the kinematic viscosity and magnetic diffusivity is $[v]=[\eta]=[\boldsymbol{u}][\boldsymbol{r}] \approx 1.5 \times 10^{19} \mathrm{~cm}^{2} \mathrm{~s}^{-1}$, the unit for specific entropy is $[s]=c_{p}=\gamma /(\gamma-1) \mathcal{R} / \mu \approx 3.5 \times 10^{8} \mathrm{~cm}^{2} \mathrm{~s}^{-2} \mathrm{~K}^{-1}$, the unit for specific enthalpy is $[h]=[\boldsymbol{u}]^{2}=10^{4} \mathrm{~km}^{2} \mathrm{~s}^{-2}$, the temperature unit is $[T]=[h] /[s] \approx 3 \times 10^{5} \mathrm{~K}$, the density unit is $[\varrho]=\Sigma_{0} /[\boldsymbol{r}] \approx 7.5 \times 10^{-13} \mathrm{~g} \mathrm{~cm}^{-3}$, the pressure unit is $[p]=(\gamma-1) / \gamma[\varrho][h] \approx 30 \mathrm{~g} \mathrm{~cm}^{-1} \mathrm{~s}^{-2}$, the unit for the mass accretion rate is $[\dot{M}]=\Sigma_{0}[\boldsymbol{u}][\boldsymbol{r}] \approx 2 \times 10^{-7} M_{\odot} \mathrm{yr}^{-1}$, the magnetic field unit is $[\boldsymbol{B}]=[\boldsymbol{u}](4 \pi[\varrho])^{1 / 2} \approx 30 \mathrm{G}$, and the unit for the magnetic vector potential is $[\boldsymbol{A}]=[\boldsymbol{B}][\boldsymbol{r}] \approx 4 \times 10^{13} \mathrm{G} \mathrm{cm}$.

Computations have been carried out in the domain $(\varpi, z) \in$ $[0,2] \times[-1,1]$, with the mesh sizes $\delta \varpi=\delta z=0.01$. In our units, this corresponds to the domain extending to $\pm 0.1 \mathrm{AU}$ in $z$ and $0.2 \mathrm{AU}$ in $\varpi$.

We choose a set of values for our model parameters such that the resulting dimensions of our system and the resulting initial profiles of the physical quantities are close to those for a standard accretion disc around a protostellar object. The stellar radius is $r_{*}=0.15$, corresponding to 3 solar radii, the disc inner radius is $\varpi_{\text {in }}=0.6$, i.e. 4 stellar radii, the disc outer radius is $\varpi_{\text {out }}=1.9$, close to the outer domain boundary, and the disc semi-thickness is $z_{0}=0.15$, i.e. equal to the stellar radius.

Further, our choice for the entropy contrast between disc and corona, $\beta_{\text {disc }}=0.005$, leads to an initial disc temperature ranging between $9000 \mathrm{~K}$ in the inner part and $900 \mathrm{~K}$ in the outer part. Real protostellar discs have typical temperatures of about a few thousand Kelvin (e.g., Papaloizou \& Terquem 1999). As turns out from our simulations, the disc temperature increases by less than a factor of 2 with time, except for the inner disc edge where the increase is much higher. The low disc temperature corresponds to a relatively high disc density. In the inner part of the disc, it is about $3 \times 10^{-10} \mathrm{~g} \mathrm{~cm}^{-3}$ initially, but increases to about $10^{-9} \mathrm{~g} \mathrm{~cm}^{-3}$ at later stages. In the outer part it is a few times $10^{-11} \mathrm{~g} \mathrm{~cm}^{-3}$.

The Shakura-Sunyaev coefficient of the turbulent disc viscosity is $\alpha_{\mathrm{SS}}=0.004$ whereas the magnetic diffusivity is $2 \times 10^{-5}$ in the corona and in the star, and enhanced to $6 \times 10^{-5}$ in the disc. The latter corresponds to $\alpha_{\mathrm{SS}}^{(\eta)} \equiv \eta_{\text {disc }} /\left(c_{\mathrm{s}, \mathrm{disc}} z_{0}\right)$, ranging between 0.004 and 0.008 in the disc midplane. With $\eta_{\text {disc }}=6 \times 10^{-5}$ (corresponding to about $9 \times 10^{14} \mathrm{~cm}^{2} \mathrm{~s}^{-1}$ ) and the disc semi-thickness $z_{0}=0.15$, the diffusion time is $t_{\text {diff }} \equiv z_{0}^{2} / \eta_{\text {disc }}=375$.

The stellar surface angular velocity is about unity (in dimensionless units), corresponding to a rotation period of around 10 days. This means that the corotation radius is $\varpi_{\mathrm{co}} \approx$ 1 in nondimensional units. The inner disc radius (which is fixed in our model) is rotating with roughly Keplerian speed, resulting in a rotation period of the inner disc edge of around 4.5 days (3 in dimensionless units).

\subsection{Disc dynamo and stellar magnetosphere}

To date, almost all models of the formation and collimation of winds and jets from protostellar accretion discs rely on an externally imposed poloidal magnetic field and ignore any field produced in the disc. This is not the case in the model developed in Paper I, which also forms the basis of the model used in the present paper. In Paper I we study outflows in connection with magnetic fields that are solely generated and maintained by a disc dynamo, resolving the disc and the star at the same time as well as assuming a non-ideal corona. There, the initial seed magnetic field is large scale, poloidal, of mixed parity, weak and confined to the disc.

However, observations of $\mathrm{T}$ Tauri stars suggest that magnetic star-disc coupling might result in a spin-down of the star due to magnetic braking by a stellar magnetosphere penetrating the disc. Magnetic fields as strong as $1 \mathrm{kG}$ and larger have been detected on T Tauri stars (e.g., Guenther et al. 1999), and there is evidence for hot and cool spots on the stellar surface.

Our present model is similar to that of Paper I except that, in addition to the disc dynamo, we also model a magnetosphere of the protostar. For the magnetosphere, we assume that in the initial state a stellar dipolar magnetic field threads the surrounding disc, leading to a contribution of $A_{\varphi}$ given by

$A_{\varphi}(\varpi, z)=\ldots+A_{\mathrm{star}} \frac{\varpi r_{*}^{2}}{r^{3}}\left(1-\xi_{\mathrm{star}}\right)$

where $r_{*}$ is the stellar radius, $r=\left(\varpi^{2}+z^{2}\right)^{1 / 2}$ is the spherical radius, and $A_{\text {star }}$ is a parameter controlling the strength of the stellar magnetosphere. Since $\varpi=r \sin \Theta$ (with $\Theta$ the colatitude), the magnetic moment is $\mu_{\mathrm{mag}}=A_{\text {star }} r_{*}^{2}$ (cf. Model I in Miller \& Stone 1997). The dots in Eq. (6) denote the contribution from the seed magnetic field in the disc. Since the magnetic field describing the magnetosphere is force-free, the hydrostatic equilibrium is not affected but equal to the magnetostatic equilibrium. $\xi_{\text {star }}$ is a smoothed profile for the star and equal to unity only at the origin (centre of the star), so that the magnetosphere extends to parts of the star as well. In order to anchor the magnetosphere within the star and on the stellar surface, $\varrho, \boldsymbol{u}$, and $\boldsymbol{A}$ are set to their initial values at each timestep in the region including the star and extending to about $1.5 r_{*}$. Therefore, in the models with magnetosphere, there is no mass sink in the star of the type discussed in Paper I. The star is resolved in that it stays within the mesh, allowing the study of the effects of a spinning dipole (cf. Sect. 2.1). Note that outside the anchoring region, the magnetosphere is not imposed and can evolve dynamically with time. In the induction equation, we do not include any term containing an external magnetic field. As a reference model we choose $A_{\text {star }}=5$, which corresponds to a stellar surface magnetic field strength of about $1 \mathrm{kG}$. The magnetic moment is then about $10^{37} \mathrm{G} \mathrm{cm}^{3}$.

\section{Results}

We consider models with magnetospheres of different strengths. We declare as our reference model one with a stellar surface field strength of $1 \mathrm{kG}$ (Sect. 3.1), and compare it 


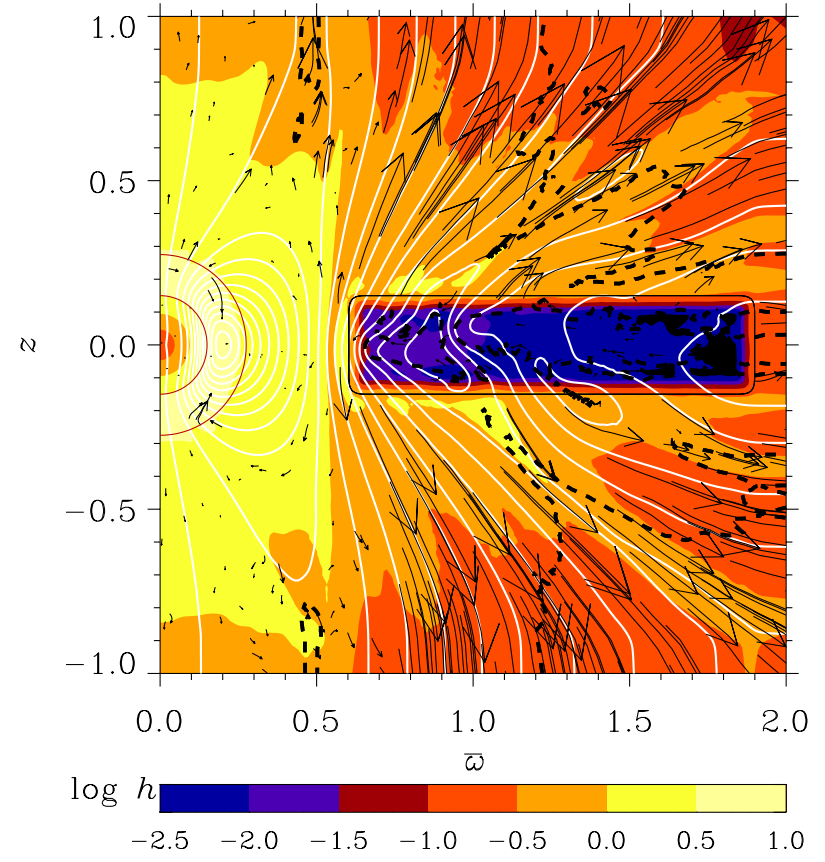

Fig. 2. Poloidal velocity vectors and poloidal magnetic field lines (white) superimposed on a colour/grey scale representation of $\log _{10} h$ for our reference model (Model M1) with a stellar surface magnetic field strength of about $1 \mathrm{kG}$ and with disc dynamo at the time $t=1267$. Specific enthalpy $h$ is directly proportional to temperature $T$, and $\log _{10} h=(-2,-1,0,1)$ corresponds to $T \approx\left(3 \times 10^{3}, 3 \times 10^{4}, 3 \times\right.$ $\left.10^{5}, 3 \times 10^{6}\right) \mathrm{K}$. The black dashed line shows the surface where the poloidal velocity equals the Alfvén speed from the poloidal magnetic field (Alfvén surface). The disc boundary is shown as a thin black line. In red are marked the stellar surface as well as the surface up to which the magnetosphere is anchored. Here and in the following figures, the field lines tend to become vertical near the boundaries, but this is due to artifacts from the boundary conditions.

with a model without disc dynamo (Sect. 3.2), and with models whose stellar surface field strength is varied between zero and $5 \mathrm{kG}$ (Sects. 3.3-3.7). Finally, the magnetic and accretion torques on the star are studied for different magnetospheric models (Sect. 3.8).

\subsection{Reference model: $\left|B_{\text {surf }}\right| \approx 1 k G$ (Model M1)}

Our simulations show that, similar to Paper I, also the present model with a stellar magnetosphere (in addition to the disc dynamo) develops a structured outflow, composed of a stellar wind and a disc wind; see Fig. 2. The disc wind is faster, cooler and less dense, with the highest velocities in the wind originating from the inner edge of the disc, whereas the stellar wind is slower, hotter and denser. The inner disc wind is magneto-centrifugally accelerated, whereas the outer disc wind and the stellar wind are mostly pressure-driven. These driving and acceleration mechanisms will be explained in Sect. 3.5, using Model S in which the outflow structure is most pronounced.

The disc wind velocity reaches about $240 \mathrm{~km} \mathrm{~s}^{-1}$, whereas the stellar wind velocity goes only up to about $10 \mathrm{~km} \mathrm{~s}^{-1}$. The disc wind mass loss rate is time-dependent with an average value of around $2 \times 10^{-7} M_{\odot} \mathrm{yr}^{-1}$.

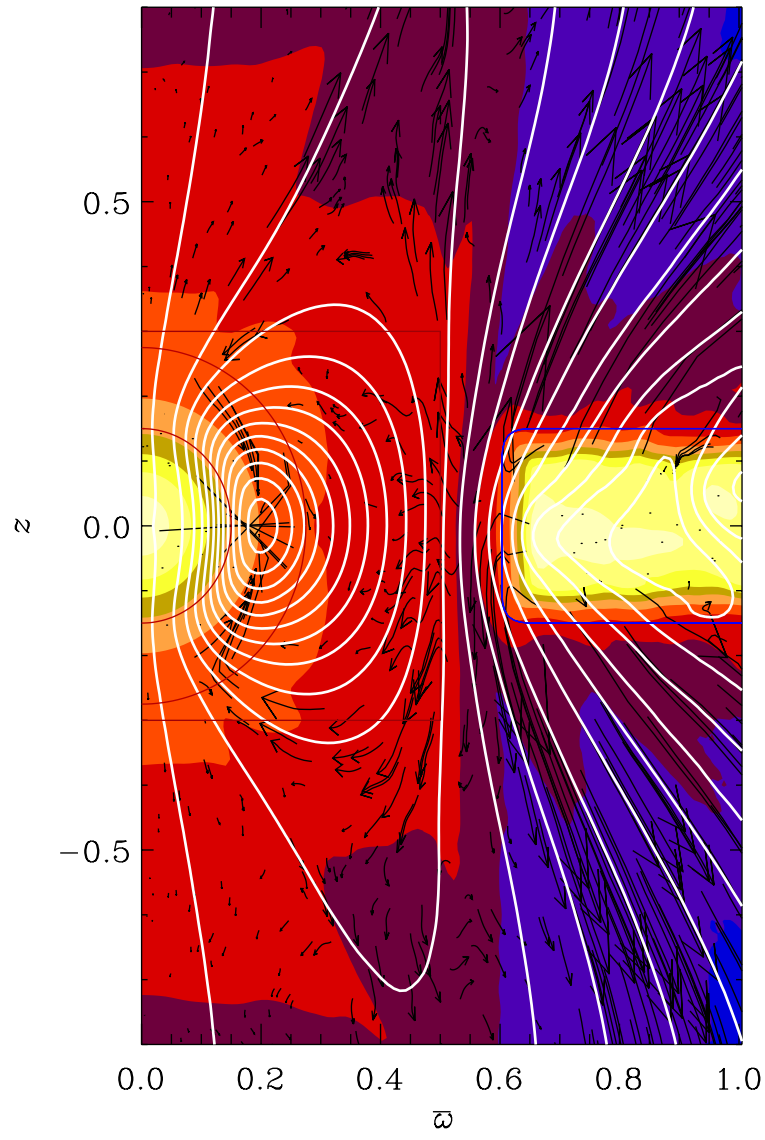

Fig. 3. Reference model (Model M1) at the time $t=1267$ when the accretion rate is maximum. Colour/grey scale representation of the density (bright colours or light shades indicate high values; dark colours or dark shades indicate low values) with poloidal magnetic field lines superimposed (white) and the azimuthally integrated mass flux density, represented as the vector $2 \pi \varpi \varrho\left(u_{\varpi}, u_{z}\right)$, shown with arrows (except in the disc where the density is high and the mass flux vectors would be too long).

Accretion of matter onto the central star is highly episodic with a maximum rate of about $4 \times 10^{-9} M_{\odot} \mathrm{yr}^{-1}$. Figure 3 shows a snapshot at a time when the accretion rate is maximum. We estimate the mass accretion rate in the following way. We put a cylinder around the axis extending to $\varpi=0.5$ and $z= \pm 0.3$. (Note that the disc inner edge is at $\varpi=0.6$, and the magnetosphere is anchored up to $r_{0} \approx 0.275$ due to a smoothed profile for the anchoring region.) Then we calculate the azimuthally integrated mass flux density, $2 \pi \varpi \varrho u$, normal to the boundaries of the cylinder and integrate it over the boundaries. We take into account that there is mass loss due to the stellar wind. It turns out that in this model, matter enters the cylinder mainly through the vertical boundary and only then partly flows along magnetospheric field lines, because the magnetosphere does not extend far enough.

\subsection{A model with no disc dynamo (Model M1-0)}

Almost all previous work on the magnetic star-disc coupling assumes that the magnetic field in the disc results entirely from the central star. Some models include an externally imposed 


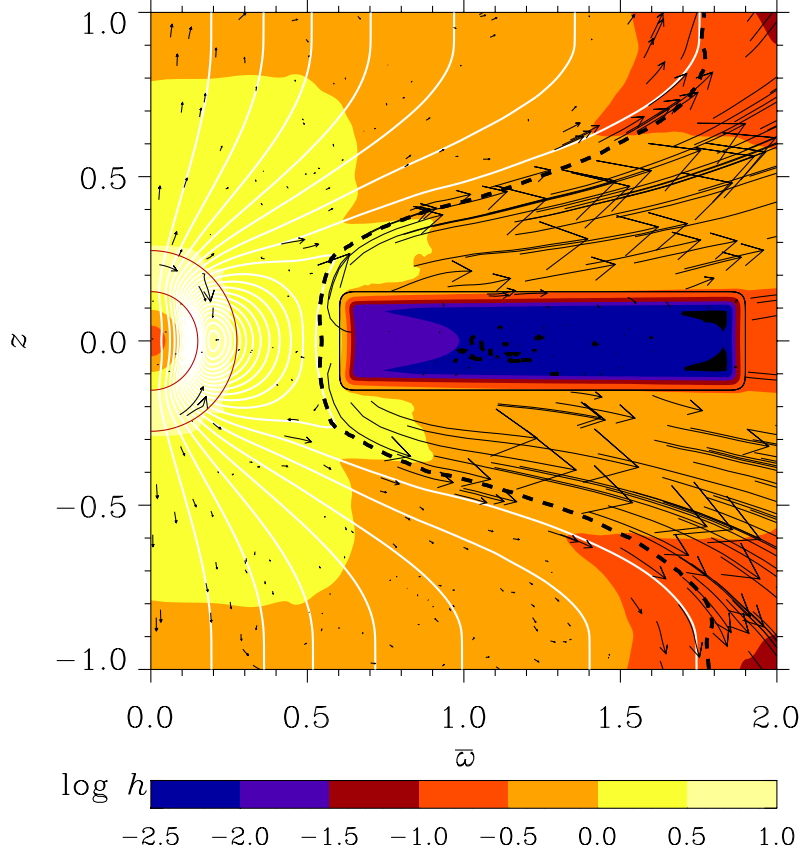

Fig. 4. Model M1-0. Same model as the reference model in Fig. 2, but with no disc dynamo, i.e. $\alpha=0$ in Eq. (3), and averaged over times $t=1100 \ldots t=1127$. Note that, unlike all the models with disc dynamo, the magnetic field (field lines shown in white) is almost entirely swept out of the disc.

magnetic field (e.g., Küker et al. 2003). In order to study the effect of the disc dynamo on the overall field structure and on the resulting outflow, we have calculated a model where the disc dynamo is turned off, i.e. we put $\alpha=0$ in Eq. (3).

As can be seen in Fig. 4, the magnetic field in the disc is now almost entirely swept away. The initial disc field due to the penetrating magnetosphere is expelled and only a very weak disc field remains without a dynamo. As a consequence, the structure and driving/acceleration mechanism of the disc wind change significantly. Transport of specific angular momentum is weak and almost entirely in the $\varpi$ direction, along very weak field lines threading the disc and running in the low corona almost parallel to the disc surface. The resulting disc wind along these field lines is therefore also mainly in the horizontal direction (Fig. 4). Since the field threading the disc is very weak, the disc wind is always super-Alfvénic, reaching a speed of about $160 \mathrm{~km} \mathrm{~s}^{-1}$, so that no magneto-centrifugal acceleration can take place. Characteristic for the models with disc dynamo is a cooler, less dense region ("conical shell") that originates from the inner disc edge, and that has higher specific angular momentum than elsewhere and contains the inner magneto-centrifugally accelerated disc wind. This conical shell disappears as the magnetic field, that was threading the disc, is swept away and no disc dynamo is generating and maintaining a strong enough field. As a result, the pressure force becomes then the only driving mechanism of the whole disc wind.

As the disc field is expelled, the size of the closed stellar magnetosphere shrinks; the extended open stellar field lines now find place to run in the corona parallel above the very weak field lines threading the disc; see Fig. 4. (Note that in

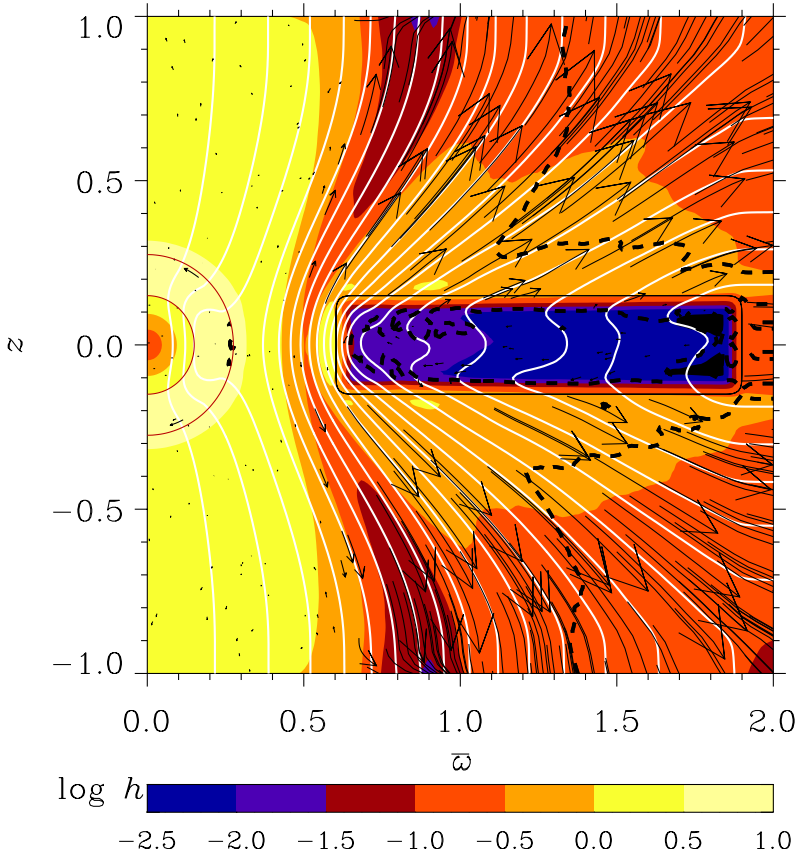

Fig. 5. Model W (weak magnetosphere and disc dynamo); the magnetic field strength at the stellar surface is about $200 \mathrm{G}$. Averaged over times $t=825 \ldots t=850$. Shown is the same as in Fig. 2 .

our magnetospheric models, the vertical alignment of the field lines near the boundaries is due to boundary conditions. In order to stabilize our code at the boundaries (especially in situations where the wind speed is low), we force matter to be slowly advected from the computational domain normal to the boundaries, rather than in spherical radial direction as in Paper I.) The stellar wind, however, does not seem to be much affected, with a maximum speed of about $10 \mathrm{~km} \mathrm{~s}^{-1}$.

The average disc wind mass loss rate does not change much in comparison with the reference model, and remains around $2 \times 10^{-7} M_{\odot} \mathrm{yr}^{-1}$. Accretion is still highly episodic; the maximum mass accretion rate is roughly 5 times larger compared to the same model but with disc dynamo, so it is now about $2 \times 10^{-8} M_{\odot} \mathrm{yr}^{-1}$.

\subsection{Weak magnetosphere: $\left|B_{\text {surf }}\right| \approx 200$ G (Model W)}

A stellar surface field strength of $200 \mathrm{G}$ is not large enough to maintain a closed magnetosphere; see Fig. 5. The field lines open up entirely to form open stellar and disc fields. This mainly affects the stellar wind; its maximum speed is reduced to about $2.5 \mathrm{~km} \mathrm{~s}^{-1}$, whereas the disc wind velocity reaches about $200 \mathrm{~km} \mathrm{~s}^{-1}$. However, the disc dynamo again produces a structure in the disc wind with different driving mechanisms. Transport of specific angular momentum is also enhanced along field lines threading the midplane at a radius smaller than the prescribed inner disc radius, which is due to an increased angular velocity. In the disc, the field lines show a characteristic wiggly pattern similar to the so-called channel flow solution seen in two-dimensional simulations of the Balbus-Hawley instability (Hawley \& Balbus 1991). This instability is indeed to be expected for the weak field strengths 


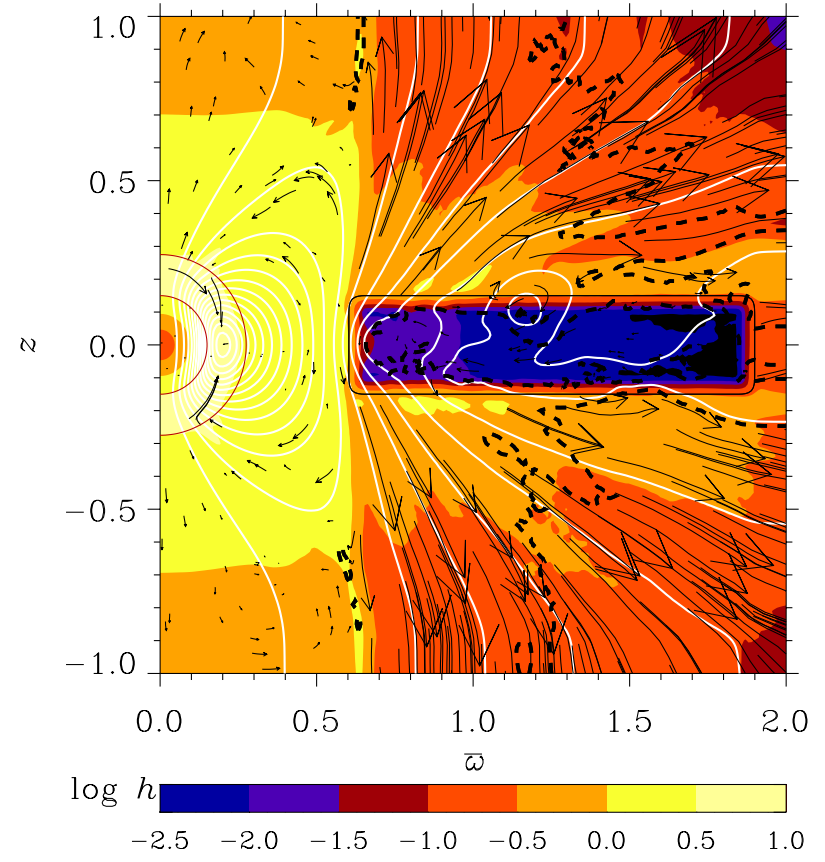

Fig. 6. Model M2 (medium magnetosphere and disc dynamo); the magnetic field strength at the stellar surface is about $2 \mathrm{kG} . t=562$. Shown is the same as in Fig. 2.

in the present model; for stronger fields this instability is suppressed. Note, however, that this channel flow type solution in the disc can only be seen in the time-averaged picture shown in Fig. 5.

In this connection it might be worthwhile reiterating that the purpose of introducing a turbulent viscosity is of course to imitate the effects of the three-dimensional Balbus-Hawley instability. The channel flow solution is clearly an artifact of two dimensions, and we regard the almost complete absence of the channel flow behaviour in our solutions as a confirmation that there is no "double-instability" of the mean flow in the sense discussed above (see also Tuominen et al. 1994 and Yousef et al. 2003 for discussions regarding the problems of stability studies of mean-field models).

\subsection{Medium magnetosphere: $\left|B_{\text {surf }}\right| \approx 2 k G$ (Model M2)}

A stellar magnetosphere with an increased stellar surface field strength of about $2 \mathrm{kG}$ interacts with the disc dynamo in such a way that the resulting magnetic field, temperature and density distributions are clearly influenced. The structure in the disc wind becomes more visible, with a more pronounced (cooler and less dense) conical shell originating from the inner disc edge containing a (slightly) faster magneto-centrifugally accelerated inner disc wind with a maximum speed of about $250 \mathrm{~km} \mathrm{~s}^{-1}$; compare Figs. 2 and 6 . The average disc wind mass loss rate stays around $2 \times 10^{-7} M_{\odot} \mathrm{yr}^{-1}$, and the stellar wind velocity does not exceed $10 \mathrm{~km} \mathrm{~s}^{-1}$.

When the stellar surface field is as strong as $2 \mathrm{kG}$, the highly episodic accretion is correlated with magnetospheric oscillations. During time intervals when the magnetosphere is

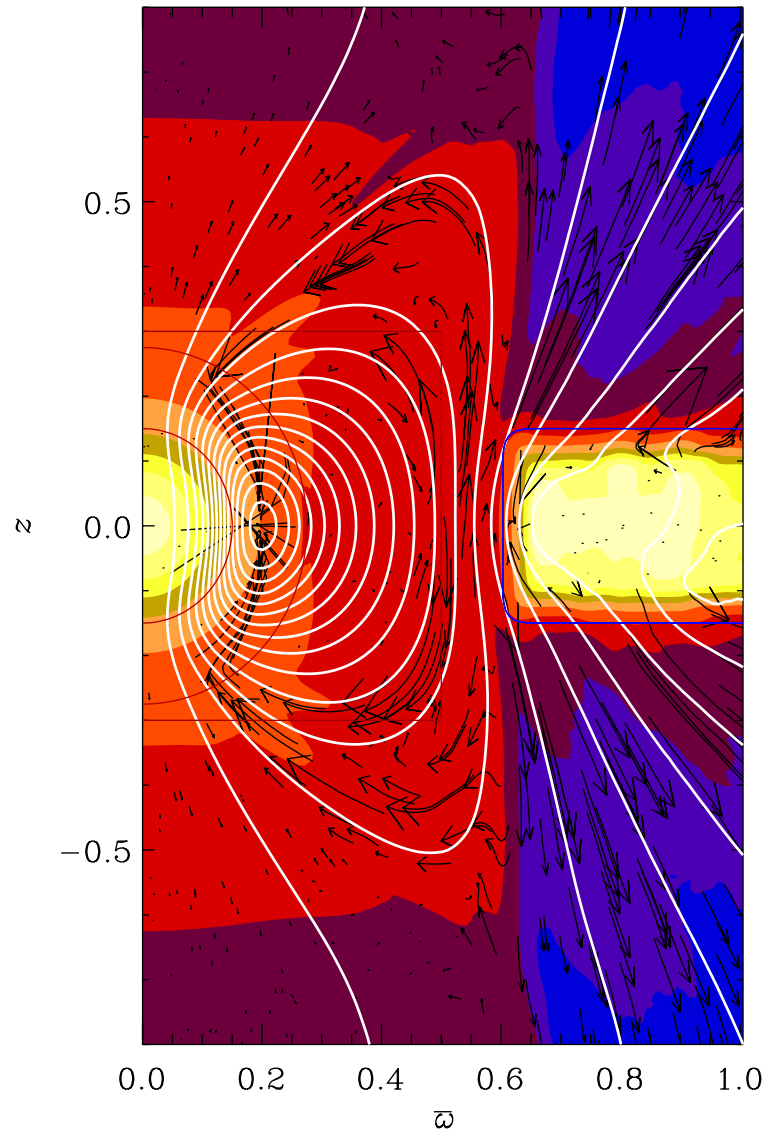

Fig. 7. Model M2. $t=562$. Shown is the same as in Fig. 3, also at a time when the accretion rate is maximum.

expanded so that the outer closed field lines are sufficiently close to the disc, the accretion flow is along magnetospheric field lines; see Fig. 7 (see also Sect. 3.5). The maximum accretion rate is about $2 \times 10^{-8} M_{\odot} \mathrm{yr}^{-1}$.

\subsection{Strong magnetosphere: $\left|B_{\text {surf }}\right| \approx 5 k G$ (Model S)}

The time sequence shown in the lower panel of Fig. 8 shows that the magnetosphere is oscillating with a period of around 15 to 30 days. As we shall show below, the oscillations mean that the magnetosphere is expanding and contracting and in this way periodically connecting and disconnecting open stellar and disc fields, therefore changing the magnetic star-disc coupling.

It turns out that the highly episodic accretion flow and accretion rate are correlated with the configuration of the magnetosphere (compare upper and lower panels of Fig. 8). Low values of $B_{z}$ at the position $(\varpi, z)=(0.6,0.4)$, correspond to a configuration where closed magnetospheric lines penetrate the inner disc edge, thus connecting the star to the disc (as in Fig. 9). In this case, disc matter is loaded onto magnetospheric field lines and flows along them to accrete onto the star. As a consequence, the accretion rate is highest during these phases, typically up to between $10^{-8} M_{\odot} \mathrm{yr}^{-1}$ and $2.5 \times 10^{-8} M_{\odot} \mathrm{yr}^{-1}$ (see Fig. 8, upper panel).

High values of $B_{z}$ at the position $(\varpi, z)=(0.6,0.4)$, correspond to a configuration when the outer field lines of the 


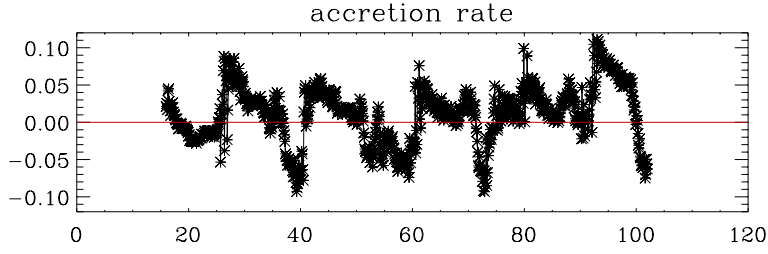

$\mathrm{B}_{\mathrm{z}}$ at $(\bar{\omega}, z)=(0.6,0.4)$

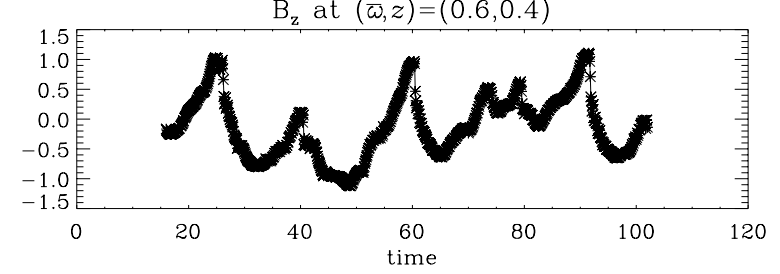

Fig. 8. Model S (strong magnetosphere and disc dynamo); the magnetic field strength at the stellar surface is about $5 \mathrm{kG}$. Mass accretion rate estimated as described in Sect. 3.1 (upper panel) and vertical magnetic field component at $(\varpi, z)=(0.6,0.4)$ (lower panel) as functions of time. The latter is an indicator for the oscillations of the magnetosphere. Note that the accretion rate and the magnetospheric oscillations are correlated such that the accretion rate is high when $B_{z}$ at the position given above is low. This corresponds to the state when the star is connected to the disc by its magnetosphere. Time is here in non-dimensional units, but with a time unit of 1.5 days, the oscillation period is seen to be around 15 to 30 days.

magnetosphere have opened up into disconnected open stellar and disc field lines, thus disconnecting the star from the disc (as in Fig. 10). In this case, matter is lost directly into the outflow and there is no net accretion of disc matter (see Fig. 8, upper panel).

However, even when the accretion rate is high, most of the mass that leaves the disc goes into the wind. The mass loss rates into the disc wind are about one order of magnitude higher compared to the peak accretion rates (see Fig. 11, upper panel), and not very different from the rates in our other magnetospheric models presented in previous subsections, ranging between $10^{-7} M_{\odot} \mathrm{yr}^{-1}$ and $2.5 \times 10^{-7} M_{\odot} \mathrm{yr}^{-1}$. The disc wind mass loss rates are fluctuating with time on rather short time scales, and no correlation with the oscillating magnetosphere can be detected (compare upper and lower panels of Fig. 11).

The time-dependent behaviour of the accretion flow and the disc wind is interesting in view of observations. Recent highresolution short- and long-term monitoring of classical T Tauri stars (CTTS) with the ESO Very Large Telescope reveals that CTTS are highly variable on time scales of minutes to several years. This variability in the emission spectra is associated with both accretion and outflow processes (Stempels \& Piskunov 2002, 2003).

As in all our models with disc dynamo, the dynamo produces a structured disc wind. However, the larger the stellar surface magnetospheric field strength is, the more pronounced is the structure. The outflow consists of (i) a slower, hotter and denser, mostly pressure-driven stellar wind; (ii) a faster, cooler and less dense outer disc wind that is also mostly pressuredriven; and (iii) a fast magneto-centrifugally accelerated inner disc wind within a clearly visible conical shell originating from the inner disc edge, that has lower temperatures and densities

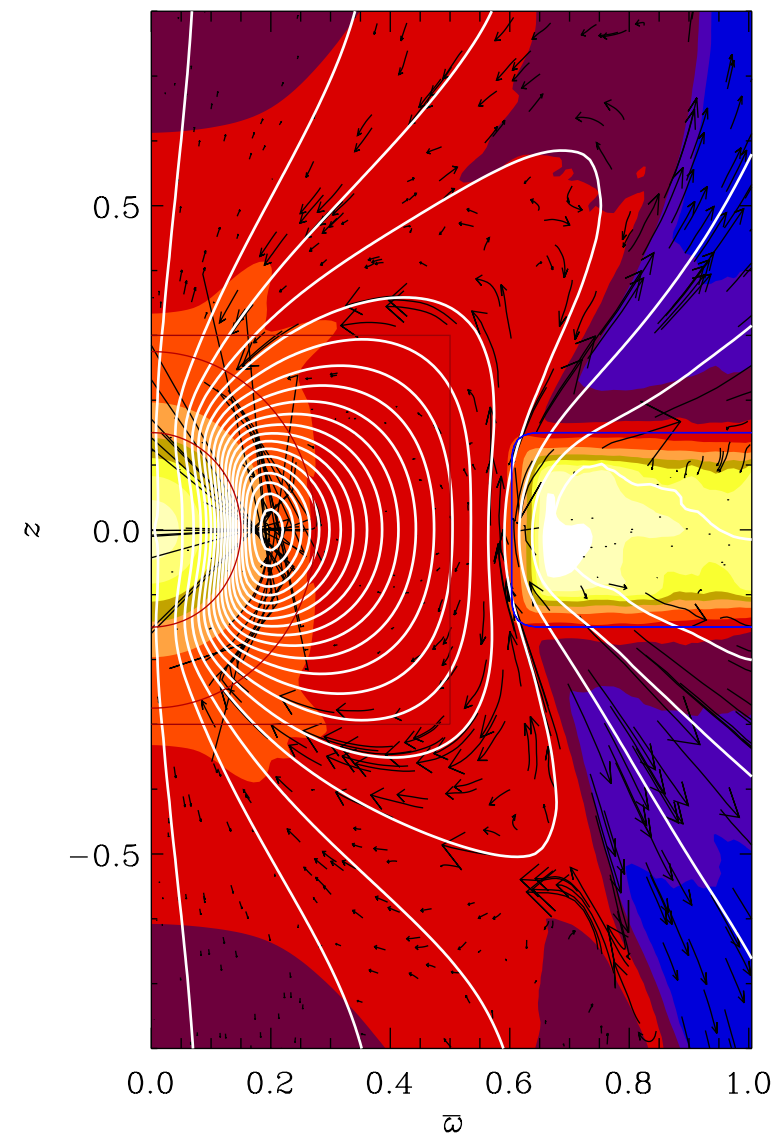

Fig. 9. Model S. Shown is the same as in Fig. 3, but at the time $t=98$ when the star is magnetically connected to the disc.

than elsewhere (see Fig. 12). This outflow structure was already discussed in Paper I in some detail.

Figure 12 shows a snapshot at a time in a transition period, when the magnetic star-disc connectivity changes. In these periods when disconnected stellar and disc field lines are about to reconnect, in addition to the above described structured outflow typical for our models with disc dynamo, there is a pressuredriven, hot and dense, but relatively fast outflow between the stellar and disc winds.

As one can see in Figs. 13 and 14, specific angular momentum is carried outwards from the disc into the corona mainly along magnetic field lines threading the innermost part of the disc. Here the dynamo-generated field is strongest and is responsible for confining the conical shell in the corona. The angle between the rotation axis and these field lines is $30^{\circ}$ and larger at the disc surface, which is favourable for magneto-centrifugal acceleration (Blandford \& Payne 1982; see Campbell 1999, 2000, 2001 for a more detailed treatment). Furthermore, in the conical shell the flow is highly supersonic but the Alfvén radius is about two times larger than the radius where the field lines have their footpoints at the disc surface. This is sufficient for the magnetic field lines to act as a lever arm along which the inner disc wind is accelerated. On the other hand, in the outer disc wind the Alfvén surface is very close to the disc surface. As a consequence, acceleration due to the pressure gradient becomes more important there, although 


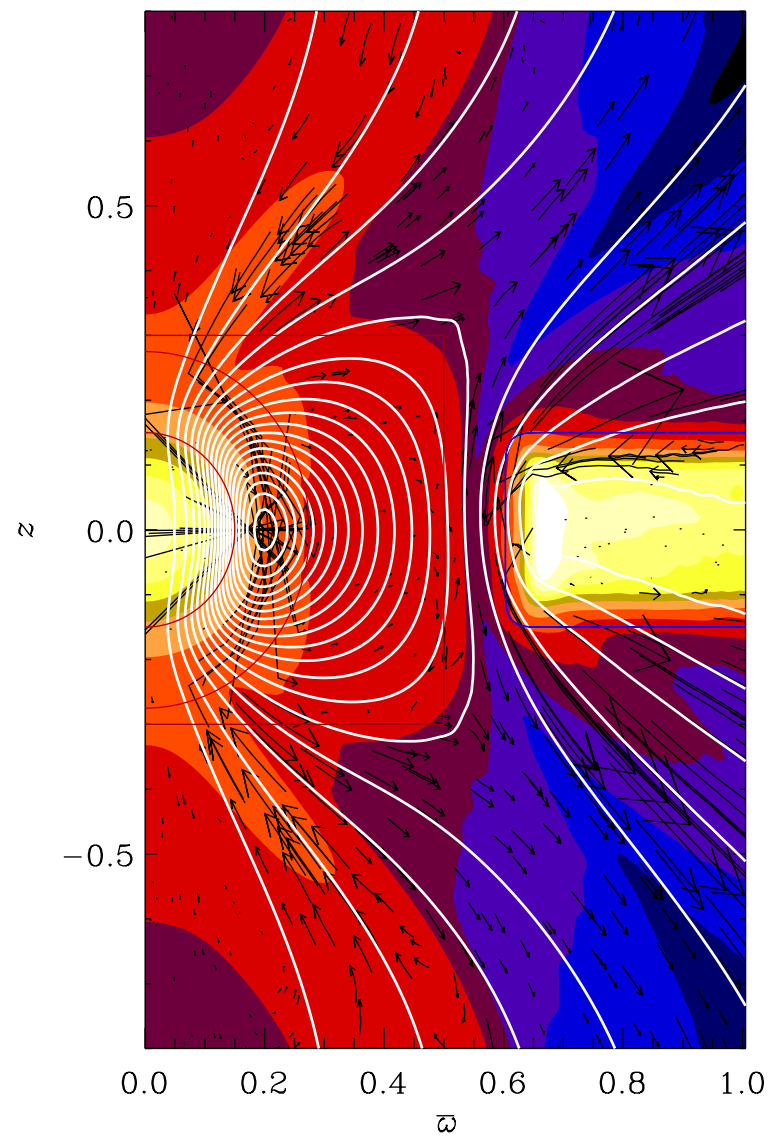

Fig. 10. Model S. Shown is the same as in Fig. 9, but at the time $t=102$ when the star is magnetically disconnected from the disc.

the criterion of Blandford \& Payne (1982) is still fulfilled. The stellar wind is mostly pressure-driven, carrying almost no specific angular momentum and remaining subsonic. The outflow between the stellar and disc winds has small azimuthal velocity and small specific angular momentum, and becomes quickly super-Alfvénic.

A confirmation of the presence of coexisting pressuredriving and magneto-centrifugal acceleration of the winds as described above, can be obtained by looking at the ratio between the poloidal magneto-centrifugal and pressure forces, $\left|\boldsymbol{F}_{\text {pol }}^{(\mathrm{mc})}\right| /\left|\boldsymbol{F}_{\text {pol }}^{(\mathrm{p})}\right|$, where the subscript "pol" denotes the poloidal components. The forces appear in the equation of motion as

$\boldsymbol{F}^{(\mathrm{mc})}=\varrho\left(\Omega^{2} \varpi-\nabla \Phi\right)+\boldsymbol{J} \times \boldsymbol{B}$,

and $\boldsymbol{F}^{(\mathrm{p})}=-\boldsymbol{\nabla} p$. Their ratio is shown in Fig. 15. Again, the conical shell can be clearly seen. In the conical shell, the ratio assumes large values which confirms that magneto-centrifugal launching and acceleration of the disc wind is dominant there. On the other hand, at the outer parts of the disc surface the pressure force is stronger, leading to pressure-driving. At the stellar surface, both forces have comparable strengths; however, in large parts of the stellar wind the pressure force becomes dominant. The outflow between the stellar and disc winds is also pressure-dominated.

Figures 13 and 14 show the distribution of specific angular momentum for the two cases, where the star is connected to and

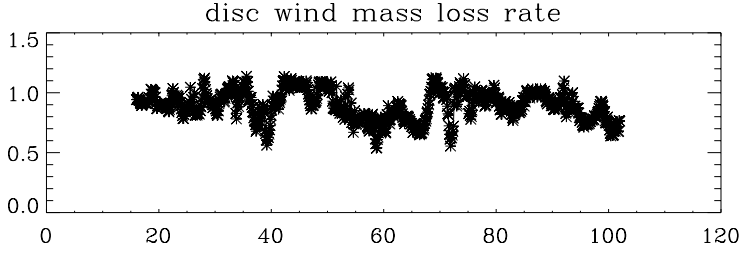

$\mathrm{B}_{\mathrm{z}}$ at $(\bar{\omega}, z)=(0.6,0.4)$

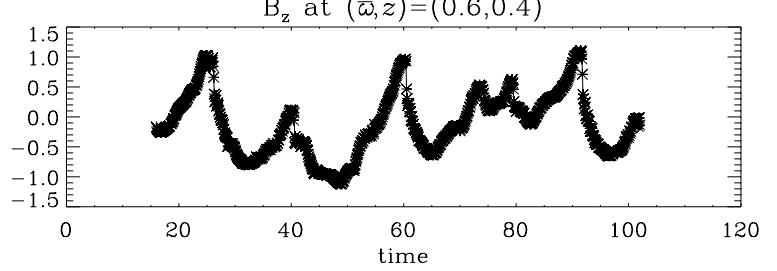

Fig. 11. Model S. Disc wind mass loss rate (upper panel) and vertical magnetic field component at $(\varpi, z)=(0.6,0.4)$ (lower panel) as functions of time. The latter is an indicator for the oscillations of the magnetosphere. No correlation between the disc wind mass loss rate and the magnetospheric oscillations can be seen.

disconnected from the disc, respectively. Comparing these two figures, one can see that in periods of no net accretion, when the star is disconnected from the disc, specific angular momentum transport by the inner disc wind is visibly enhanced, carried by enhanced stellar and inner disc winds. Their velocities are larger in these periods: terminal outflow speeds are then about $20 \mathrm{~km} \mathrm{~s}^{-1}$ in the stellar wind and about $400 \mathrm{~km} \mathrm{~s}^{-1}$ in the conical shell of the disc wind, where velocities are highest. Accretion flow velocities in the disc are up to about $15 \mathrm{~km} \mathrm{~s}^{-1}$. In our computational domain, both the inner stellar wind and the funnel flow remain subsonic at all times. The slow stellar wind is likely to be due to our boundary conditions that we impose in our magnetospheric models by fixing the poloidal velocity to be zero in the star.

A peculiar feature of all our models with a disc as cool as in the models of this paper, is the presence of at least one radial polarity reversal of the dynamo-generated magnetic field in the disc (cf. Figs. 23-25 of Paper I). In the model with strong magnetosphere discussed in this section, the first reversal occurs roughly at the corotation radius $\left(\varpi_{\mathrm{co}} \approx 1\right)$, where the angular velocity is about $7.5 \times 10^{-6} \mathrm{~s}^{-1}$, corresponding to a rotation period of around 10 days.

The results in Sects. 3.4 and 3.5 concerning the oscillating magnetosphere and associated reconnection processes and episodic accretion, look indeed like being in the regime described by Goodson \& Winglee (1999). However, our stellar wind is relatively slow and mostly pressure-driven whereas their stellar wind (jet) is fast and magneto-centrifugally driven. On the other hand, the main new feature induced by our disc dynamo is the structure in our disc wind. Whereas the outer disc wind is slower and mostly pressure-driven, the inner disc wind is fast, and launched and accelerated magnetocentrifugally; it might collimate at larger heights to form the observed protostellar jet. The magnetospheric extension is limited by the dynamo-generated disc field that is strongest in the inner disc parts and advected into the disc corona. 


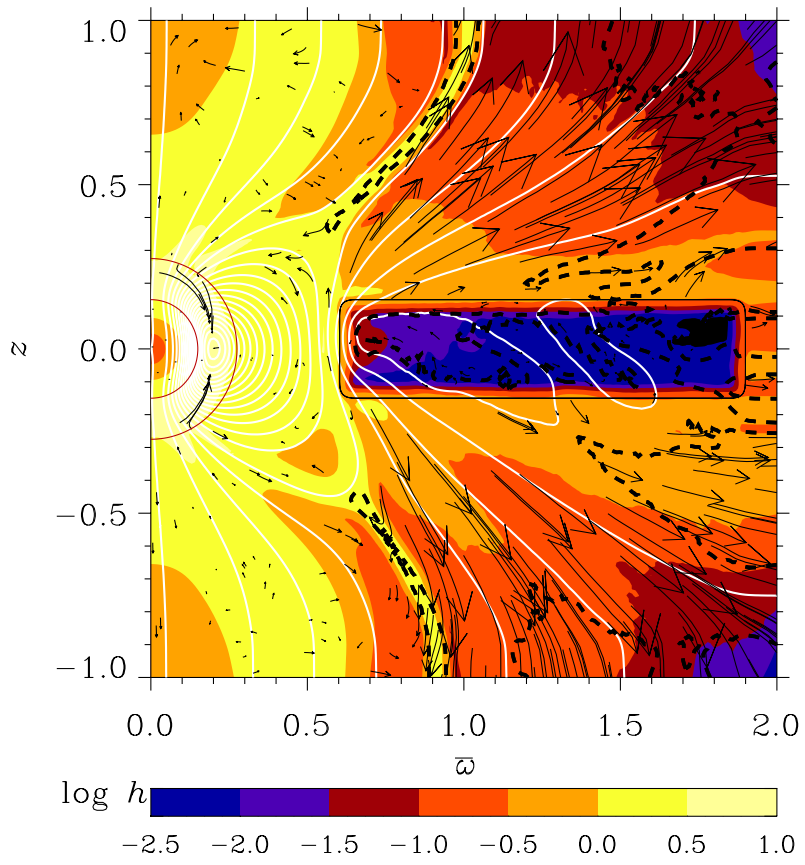

Fig. 12. Model S. $t=103$ when the star is about to be magnetically reconnected to the disc. Shown is the same as in Fig. 2.

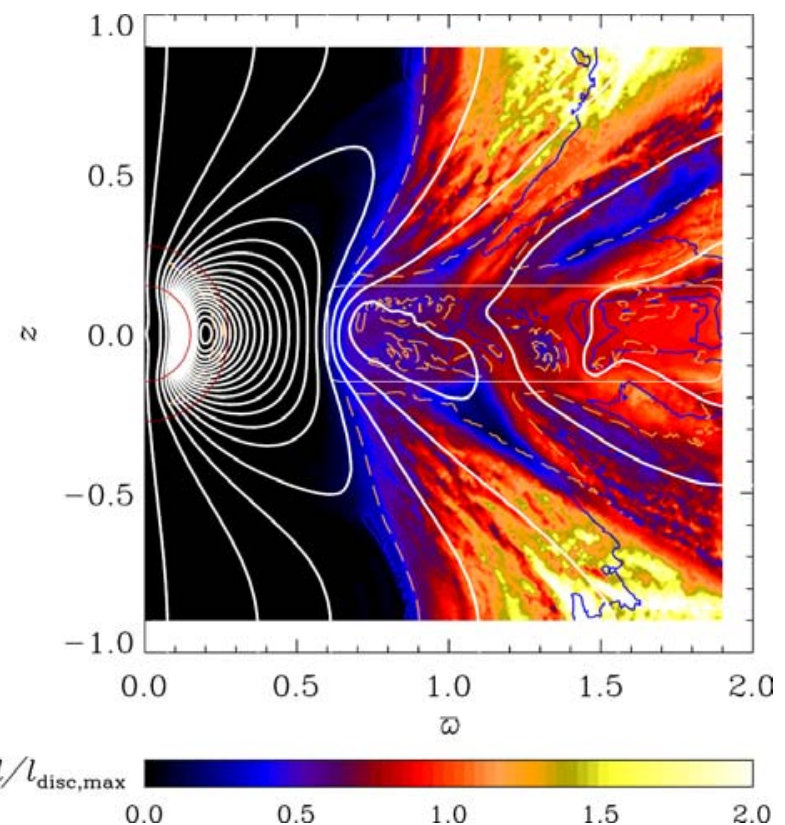

Fig. 13. Colour/grey scale representation of the specific angular momentum, $l=\varpi u_{\varphi}=\varpi^{2} \Omega$, normalized by the maximum specific angular momentum in the disc, $l_{\text {disc,max }}$, with poloidal magnetic field lines superimposed (white) for Model S at the time $t=98$ when the star is magnetically connected to the disc. The blue solid line shows the Alfvén surface, and the orange dashed line the sonic surface.

\subsection{Dependence on stellar field strength}

In Table 1 we summarize the parameters that have been changed in the different models considered in this paper and that are related to the physics of both the magnetosphere and the disc dynamo. The strength of the stellar field is given both in nondimensional and in dimensional units, together with the

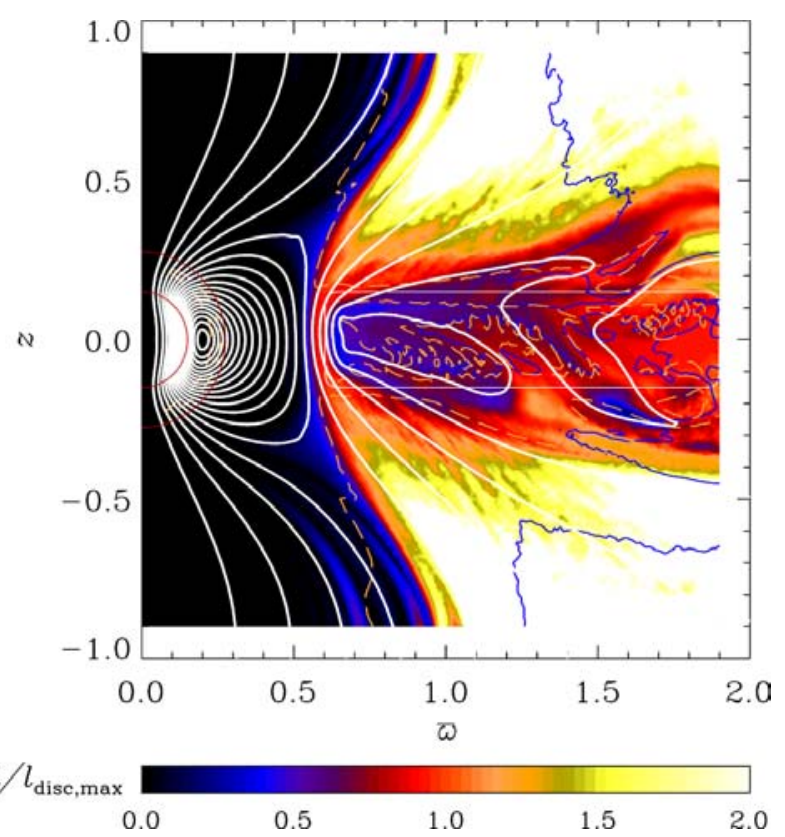

Fig. 14. Same as in Fig. 13 (Model S), but at the time $t=102$ when the star is magnetically disconnected from the disc. Note that now a lot of specific angular momentum leaves the disc from its inner edge.

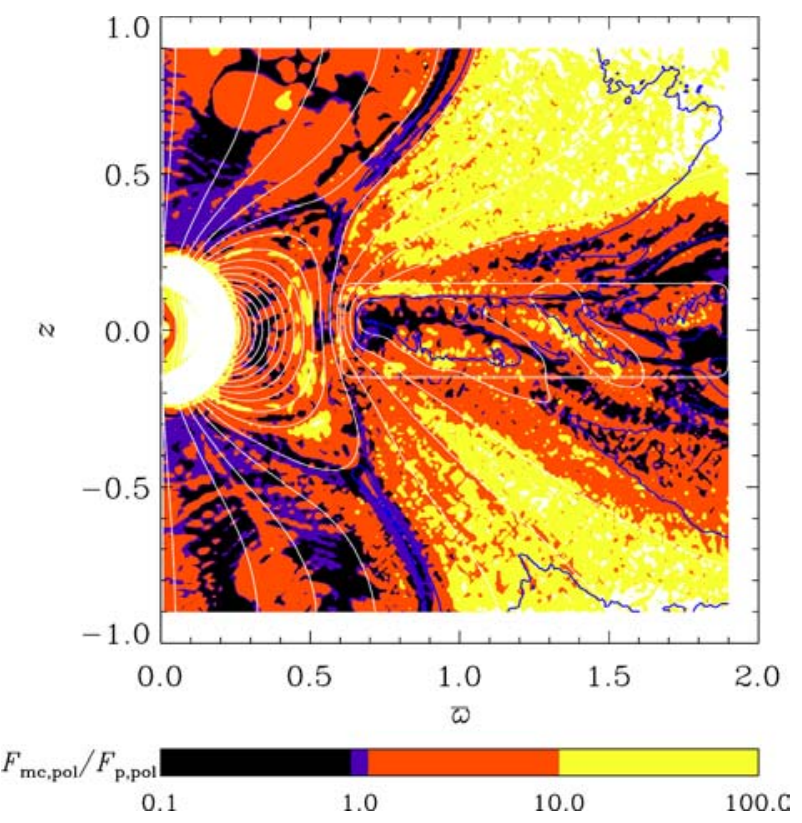

Fig. 15. Colour/grey scale representation of the ratio between the poloidal magneto-centrifugal and pressure forces, $\left|\boldsymbol{F}_{\mathrm{pol}}^{(\mathrm{mc})}\right| /\left|\boldsymbol{F}_{\mathrm{pol}}^{(\mathrm{p})}\right|$ as defined in Eq. (7) and below, with larger values corresponding to lighter shades. Superimposed are the poloidal magnetic field lines (white). The blue solid line shows the Alfvén surface. Model $\mathrm{S}$ at the time $t=103$ when the star is about to be magnetically reconnected to the disc.

$\alpha_{0}$ coefficient quantifying the strength of the $\alpha$ effect $\left(\alpha_{0}<0\right.$ means that $\alpha$ is negative in the upper disc plane).

As the strength of the stellar field is reduced, the size of the stellar magnetosphere shrinks. At the same time, the field in the disc also decreases somewhat. Although the conical structure becomes more pronounced as the strength of the stellar 


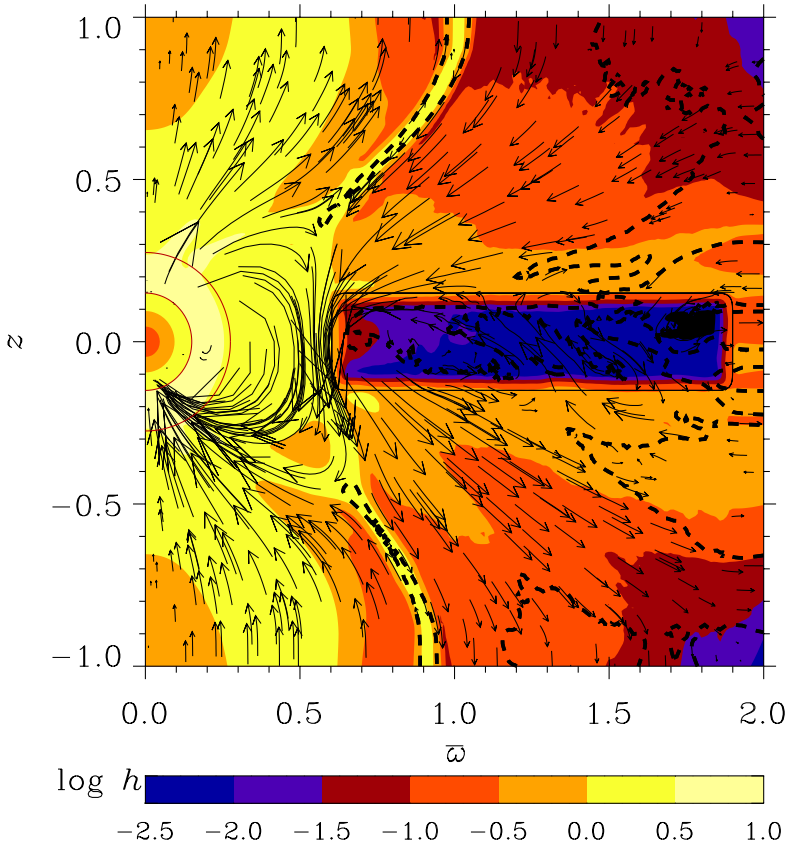

Fig. 16. Poloidal magnetic field vectors of the model shown in Fig. 12. Note that the magnetic field reversal in the corona indicates the presence of a current sheet between the magnetosphere and the field threading the disc at about $45^{\circ}$. This means that no X-point forms. The magnetic field vectors are not shown in the anchoring region. The length of the vectors is weighed with $\varpi$. The black dashed line shows the Alfvén surface.

Table 1. Summary of parameter values related to the physics of both the magnetosphere and the disc dynamo for a sequence of models where the strength of the stellar field is varied. The hyphen in the row for M1-0 indicates that there is no turbulence in that run. Note that $\left|B_{\text {surf }}\right|=A_{\text {star }} / r_{*} \times[\boldsymbol{B}]$.

\begin{tabular}{lccc}
\hline \hline Model & $A_{\text {star }}$ & $\left|B_{\text {surf }}\right|[\mathrm{kG}]$ & $\alpha_{0}$ \\
\hline $\mathrm{N}$ & 0 & 0 & -0.15 \\
$\mathrm{~W}$ & 1 & 0.2 & -0.1 \\
$\mathrm{M} 1$ & 5 & 1 & -0.1 \\
$\mathrm{M} 1-0$ & 5 & 1 & 0 \\
$\mathrm{M} 2$ & 10 & 2 & -0.1 \\
$\mathrm{~S}$ & 25 & 5 & -0.1 \\
\hline
\end{tabular}

field is increased, the overall magnetic field and outflow structures remain the same for medium and strong stellar surface magnetospheric field strengths. In particular, the field structure (inside the corotation radius) is not of X-point topology, in contrast to what is described by Shu et al. (1994). Instead, the dynamo-generated magnetic field in the disc always arranges itself such that, in the corona, the field threading the disc is anti-aligned with the stellar dipole. This necessarily leads to magnetospheric current sheets; see Fig. 16.

\subsection{A model with no magnetosphere (Model N)}

In this section we present a model without stellar magnetosphere where the only source of magnetic fields is the dynamo

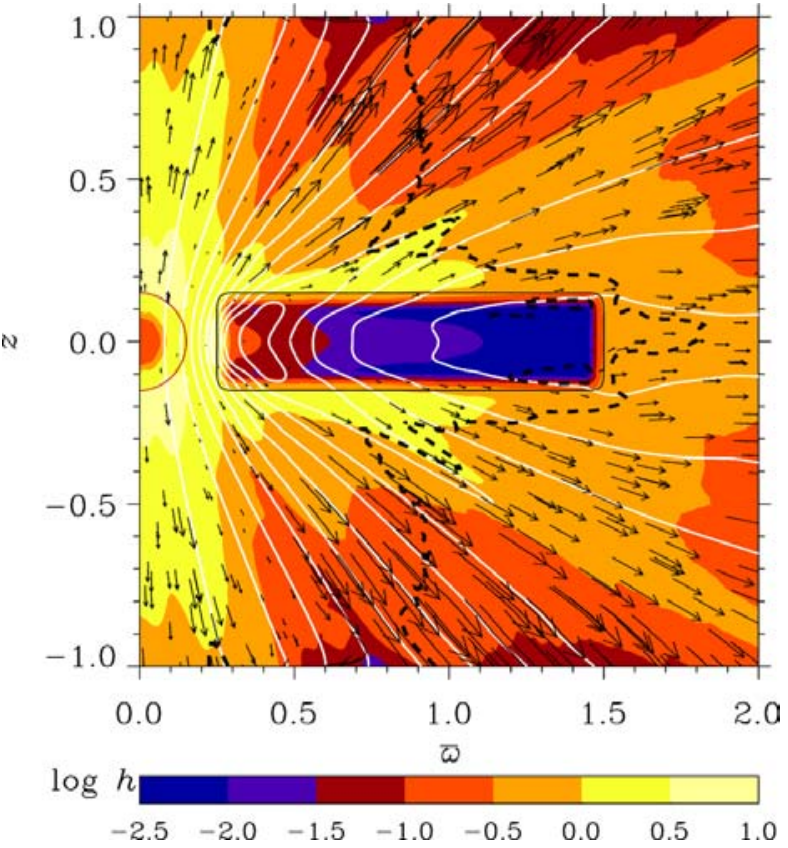

Fig. 17. Similar to Fig. 2, but for the same model as in Fig. 25 of Paper I, where now we have averaged over later times $t=584 \ldots$ $t=590$, when the disc dynamo is saturated. This corresponds to Model N (no magnetosphere and disc dynamo) in this paper. (In this figure, the black dashed line shows the surface where the poloidal velocity equals $\left(c_{\mathrm{s}}^{2}+v_{\mathrm{A}, \mathrm{pol}}^{2}\right)^{1 / 2}$, with $c_{\mathrm{s}}$ the sound speed and $v_{\mathrm{A} \text {,pol }}$ the Alfvén speed from the poloidal magnetic field.) Note the similarity of the overall outflow and coronal magnetic field structures to Model S; Fig. 12.

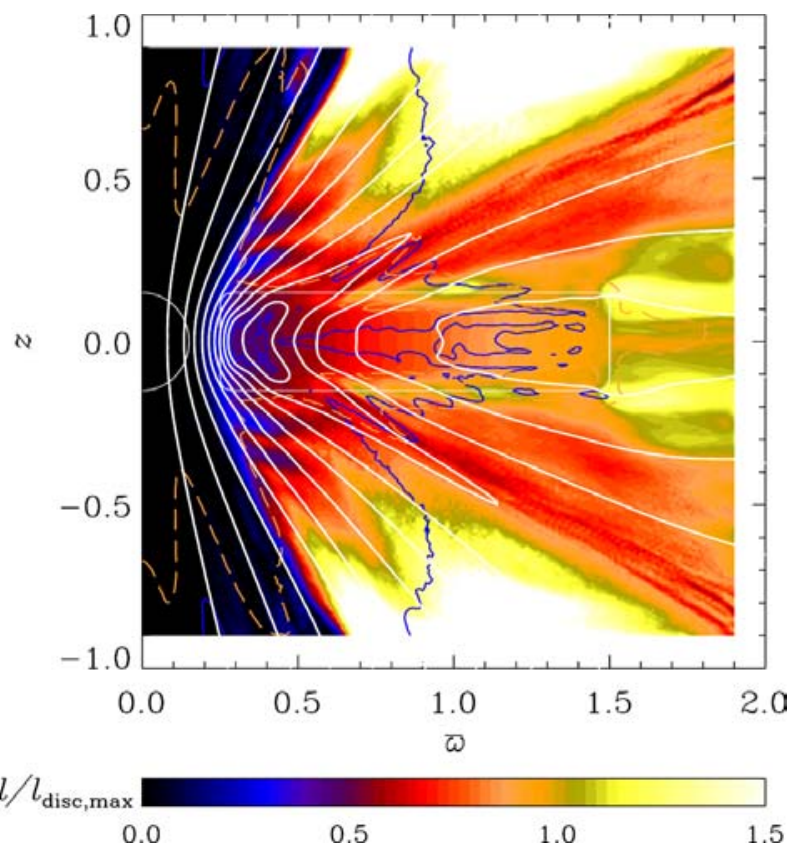

Fig. 18. Shown is the same as in Figs. 13 and 14, but for the same model (Model N) and time as in Fig. 17.

operating in the disc. The field threading the star results entirely from advection of the dynamo-generated disc field and is therefore maintained by the disc dynamo. In this model without magnetosphere, there are no restrictions to the magnetic field in 
the star. Figure 17 shows a time-averaged picture of the same model as in Fig. 25 of Paper I, but at later times, when the disc dynamo is saturated. Note that in this model the inner and outer disc radii are smaller than in the models with magnetosphere.

The overall outflow and coronal magnetic field structures are very similar to Model S (Fig. 12), as are the driving mechanisms, cf. Fig. 18 with Figs. 13 and 14. However, as one can see in Figs. 17 and 18, here the stellar wind is much faster and becomes supersonic; in contrast to Model S, in this model without magnetosphere the velocity can freely evolve in the star, and the only prescription for the star is to act as a self-regulatory mass sink (cf. Sect. 2.1). The terminal stellar wind velocity is about $150 \mathrm{~km} \mathrm{~s}^{-1}$, and the terminal inner disc wind velocity in the conical shell is also higher (about $500 \mathrm{~km} \mathrm{~s}^{-1}$ ).

Accretion is not episodic as in the magnetospheric models but the accretion rate is always positive (around $10^{-6} M_{\odot} \mathrm{yr}^{-1}$ ), and the accretion flow is in the cylindrical radial direction. The disc wind mass loss rate is around $4 \times 10^{-7} M_{\odot} \mathrm{yr}^{-1}$. Both rates are therefore higher than the rates in the models with magnetosphere. The disc wind mass loss rate is only about twice as large, but the accretion rate is at least 50 times larger, compared to when the episodic accretion in the magnetospheric models reaches its maximum rate. This changes the ratio between mass accretion rate and disc wind mass loss rate not only quantitatively, but also qualitatively. This ratio is now about 7:3, but it was less than its reciprocal value (about 1:9 at most) in the models with magnetosphere.

Nevertheless, the fact that the stellar magnetic field is still able to shield a relatively large part of the accretion flow and deflect it into the wind could be the result of the assumption of fully axisymmetric flows. Actual T Tauri stars possess highly nonaxisymmetric, non-dipolar fields (Johns-Krull et al. 1999; Johns-Krull \& Valenti 2001). However, the ratio of accretion rate to wind mass loss rate is difficult to determine observationally with great confidence. Although Pelletier \& Pudritz (1992) estimate that only about $10 \%$ of the matter joins the wind, our ratios lie still in the range of observationally derived ratios, even those for our models with magnetosphere.

\subsection{Magnetic and accretion torques}

In an axisymmetric system, the equation of conservation of angular momentum reads

$\frac{\partial}{\partial t}(\varrho l)=-\nabla \cdot\left(\boldsymbol{t}_{\mathrm{acc}}+\boldsymbol{t}_{\mathrm{mag}}+\boldsymbol{t}_{\mathrm{visc}}\right)$,

where $l=\varpi u_{\varphi}=\varpi^{2} \Omega$ is the specific angular momentum,

$\boldsymbol{t}_{\mathrm{acc}}=\varrho \varpi \boldsymbol{u}_{\mathrm{pol}} u_{\varphi}=\varrho \boldsymbol{u}_{\mathrm{pol}} \varpi^{2} \Omega$

is the material stress ( $\boldsymbol{u}_{\mathrm{pol}}$ is the poloidal velocity field),

$\boldsymbol{t}_{\mathrm{mag}}=-\varpi \frac{\boldsymbol{B}_{\mathrm{pol}} B_{\varphi}}{4 \pi}$

is the magnetic stress ( $\boldsymbol{B}_{\mathrm{pol}}$ is the poloidal magnetic field), and

$\boldsymbol{t}_{\mathrm{visc}}=-\varrho v_{\mathrm{t}} \varpi^{2} \nabla \Omega$ is the viscous stress ( $v_{\mathrm{t}}$ is the turbulent kinematic viscosity). At any given time, the total torque $T$ acting on a central object (star) is given by the volume integral

$T=-\int \boldsymbol{\nabla} \cdot\left(\boldsymbol{t}_{\mathrm{acc}}+\boldsymbol{t}_{\mathrm{mag}}+\boldsymbol{t}_{\mathrm{visc}}\right) \mathrm{d} V$,

where the integral has to be taken over a volume enclosing the star. This is equivalent to the surface integral

$$
\begin{aligned}
T & =-\oint\left(\boldsymbol{t}_{\mathrm{acc}}+\boldsymbol{t}_{\mathrm{mag}}+\boldsymbol{t}_{\mathrm{vicc}}\right) \cdot \mathrm{d} \boldsymbol{S} \\
& =\oint\left(-\varrho \boldsymbol{u}_{\mathrm{pol}} \varpi^{2} \Omega+\varpi \frac{\boldsymbol{B}_{\mathrm{pol}} B_{\varphi}}{4 \pi}+\varrho v_{\mathrm{t}} \varpi^{2} \nabla \Omega\right) \cdot \mathrm{d} \boldsymbol{S},
\end{aligned}
$$

where $\mathrm{d} \boldsymbol{S}$ is the outward directed surface element. In other words, the total torque acting on a star is given by the angular momentum flux across a surface enclosing the star (see Ghosh \& Lamb 1979b). If the flux is negative, i.e. towards the star, then the torque on the star is positive.

We calculate the angular momentum flux towards the starcarried by the matter $\left(T_{\text {acc }}\right)$, by the magnetic field $\left(T_{\text {mag }}\right)$, and due to the viscous stress $\left(T_{\text {visc }}\right)$ - across spheres around the star, so that we can write in spherical polar coordinates $(r, \Theta, \varphi)$ (with $\Theta$ the co-latitude, $\varpi=r \sin \Theta$ and $\boldsymbol{e}_{r}$ the radial unit vector):

$$
\begin{aligned}
T_{\text {acc }}(r) & =-2 \pi r^{2} \int_{0}^{\pi}\left(\boldsymbol{t}_{\mathrm{acc}} \cdot \boldsymbol{e}_{r}\right) \sin \Theta \mathrm{d} \Theta \\
& =-2 \pi r^{2} \int_{0}^{\pi} \varrho u_{r} \varpi^{2} \Omega \sin \Theta \mathrm{d} \Theta
\end{aligned}
$$

for the accretion torque,

$$
\begin{aligned}
T_{\mathrm{mag}}(r) & =-2 \pi r^{2} \int_{0}^{\pi}\left(\boldsymbol{t}_{\mathrm{mag}} \cdot \boldsymbol{e}_{r}\right) \sin \Theta \mathrm{d} \Theta \\
& =2 \pi r^{2} \int_{0}^{\pi} \varpi \frac{B_{r} B_{\varphi}}{4 \pi} \sin \Theta \mathrm{d} \Theta
\end{aligned}
$$

for the magnetic torque, and

$$
\begin{aligned}
T_{\mathrm{visc}}(r) & =-2 \pi r^{2} \int_{0}^{\pi}\left(\boldsymbol{t}_{\mathrm{visc}} \cdot \boldsymbol{e}_{r}\right) \sin \Theta \mathrm{d} \Theta \\
& =2 \pi r^{2} \int_{0}^{\pi} \varrho v_{\mathrm{t}} \varpi^{2} \frac{\partial \Omega}{\partial r} \sin \Theta \mathrm{d} \Theta
\end{aligned}
$$

for the viscous torque. In our units, $T_{\text {[acc, mag, visc] }}=1$ corresponds to about $2.5 \times 10^{38} \mathrm{erg}$.

At each position between the star and the disc where magnetic field lines are connected to the star, $B_{r} B_{\varphi}>0$ means that those field lines are leading the star in a rotational sense, therefore spinning the star up. Therefore, $T_{\mathrm{mag}}>0$ translates into a magnetic spin-up of the star. Conversely, $B_{r} B_{\varphi}<0$ means that those field lines are lagging behind the star and are therefore spinning the star down, so $T_{\mathrm{mag}}<0$ translates into a magnetic spin-down of the star.

However, $-u_{r} \varpi \Omega>0$ means only that angular momentum is added to the star. Whether or not this also means a stellar spin-up (or spin-down if $-u_{r} \varpi \Omega<0$ ), depends on the 

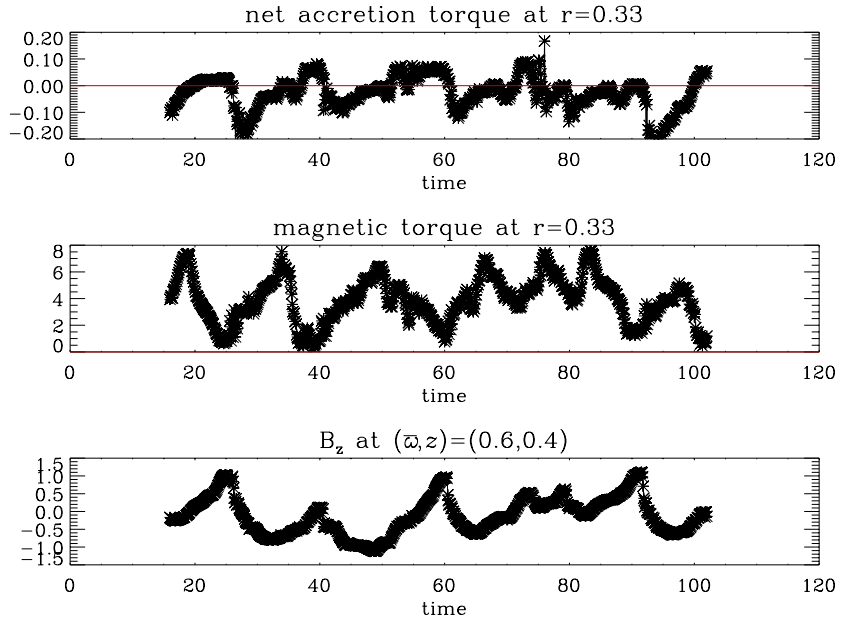

Fig. 19. Dependence of the net accretion torque $T_{\text {acc,net }}$ (upper panel) and the magnetic torque $T_{\mathrm{mag}}$ (middle panel) on time at the spherical radius $r \approx 0.33$ (close to the star) for Model S. Lower panel: same indicator for the magnetospheric oscillations as in Fig. 8, lower panel, and Fig. 11, lower panel.

magnitude of the angular velocity of the matter, $\Omega(r, \Theta)$, compared to the magnitude of the effective rotation rate of the stellar surface, $\Omega_{*}$ (see Appendix A for a precise definition). We therefore define the net accretion torque,

$T_{\text {acc,net }}(r)=-2 \pi r^{2} \int_{0}^{\pi} \varrho u_{r} \varpi^{2}\left(\Omega-\Omega_{*}\right) \sin \Theta \mathrm{d} \Theta$.

If the accreting matter $\left(u_{r}<0\right)$ is rotating faster than the star, the star will be spun up and $T_{\text {acc,net }}>0$. Likewise, if the accreting matter is rotating slower than the star, the star will be spun down and $T_{\text {acc,net }}<0$. On the other hand, a stellar wind $\left(u_{r}>0\right)$ that is rotating faster than the star, will spin down the star, and $T_{\text {acc,net }}<0$, while a stellar wind that is rotating slower than the star, will spin up the star, so $T_{\text {acc,net }}>0$.

Figure 19 shows that in Model $S$, close to the star where magnetic field lines are connected to the star, the net accretion torque is fluctuating around zero, whereas the magnetic torque is positive at all times. The magnetic torque is always much larger than the sum of the net accretion and viscous torques ( $T_{\text {visc }}$ is not shown), suggesting a total spin-up of the star, the angular momentum flux towards the star being mainly carried by the magnetic field. A correlation is clearly visible between $T_{\text {mag }}$ and the magnetospheric oscillations, and therefore also between $T_{\text {mag }}$ and the mass accretion rate. When the star is connected to the disc by its magnetosphere, i.e. when $B_{z}$ is lowest, both the accretion rate and the magnetic spin-up torque are maximum.

The peak values of the magnetic spin-up torque are around $2 \times 10^{39} \mathrm{erg}$, while those of the material spin-up torque are around $2.5 \times 10^{37} \mathrm{erg}$, and those of the material spin-down torque are around $5 \times 10^{37} \mathrm{erg}$.

Also Romanova et al. (2002) find that most of the angular momentum flux to the star is carried by the magnetosphere. In the case of a protostar rotating with a period of around 9.4 days and a stellar surface field strength of about $1.1 \mathrm{kG}$, they find

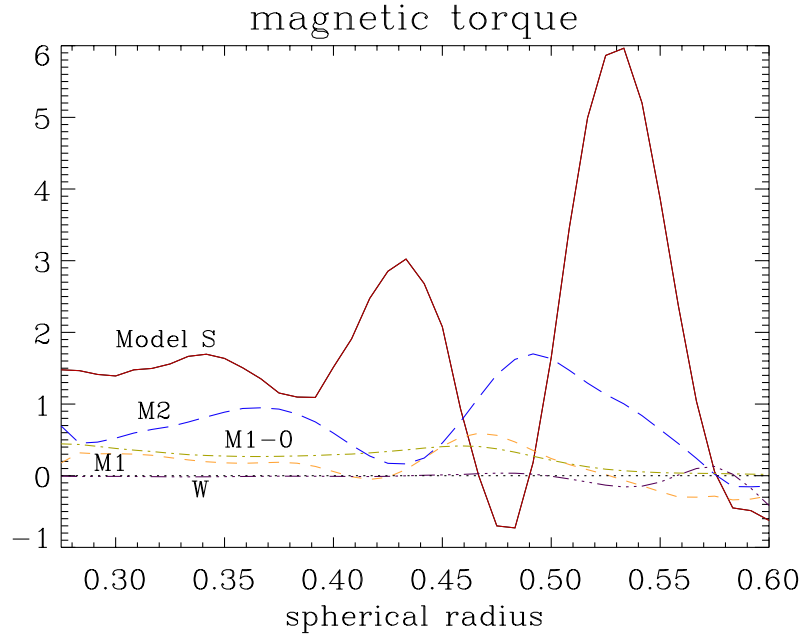

Fig. 20. Magnetic torque $T_{\mathrm{mag}}$ as function of spherical radius $r$ for the magnetospheric models presented in this paper. $T_{\text {mag }}$ is shown for radii between the approximate anchoring radius $r_{0}=0.275$ and the inner disc radius $\varpi=0.6$. Purple dash-triple-dotted: Model W, averaged over times $t=825 \ldots t=850$; orange dashed: Model M1, $t=1267$; green dash-dotted: Model M1-0, averaged over times $t=1100 \ldots t=1127$; blue long-dashed: Model M2, $t=562$; red solid: Model S, $t=103$. The black dotted line marks the magnetic torqueless state $\left(T_{\mathrm{mag}}=0\right)$.

that matter carries only about $1 \%$ of the total flux. The magnetic torque is positive so that it acts to spin up the star, and its amplitude is also correlated with the accretion rate.

In all our models with sufficiently strong magnetosphere $\left(\left|B_{\text {surf }}\right| \geq 1 \mathrm{kG}\right.$; Models M1, M1-0, M2 and S), $T_{\text {mag }}$ is mainly positive between the anchoring region and the inner disc edge $(0.275<\varpi<0.6)$; see Fig. 20 . This is roughly the region where magnetic lines are connected to the star, so that the star experiences the torque. This indicates that for sufficiently strong magnetosphere, angular momentum will be added to the star, carried by the magnetic field, resulting in a stellar spin-up by the magnetic field. As the stellar surface field strength of the magnetosphere increases, also the magnetic torque at radii between star and disc generally increases. In this region, the accretion and viscous torques (not shown) are negligible compared to the magnetic torque in all our magnetospheric models.

Figure 21 shows that when star and disc are magnetically connected, the magnetic spin-up torque is higher at all spherical radii between the approximate anchoring radius $r_{0}=0.275$ and the inner disc radius $\varpi=0.6$. Basically what happens is this: when the star is connected to the disc, there is accretion along magnetospheric field lines, and a large positive magnetic torque leads to a magnetic spin-up of the star. Then the star disconnects from the disc, and the inner disc edge is magnetically spun up (see Fig. 21); an enhanced inner disc wind carries away excess specific angular momentum (see also Fig. 14).

The latitudinal dependencies of the torques for Model $\mathrm{S}$, shown in Fig. 22, reveal that there is also a correlation between the net accretion torque (i.e. the non-integrated quantity of Eq. (21)) and the mass accretion rate. At latitudes of accretion onto the star, the net accretion torque is negative 


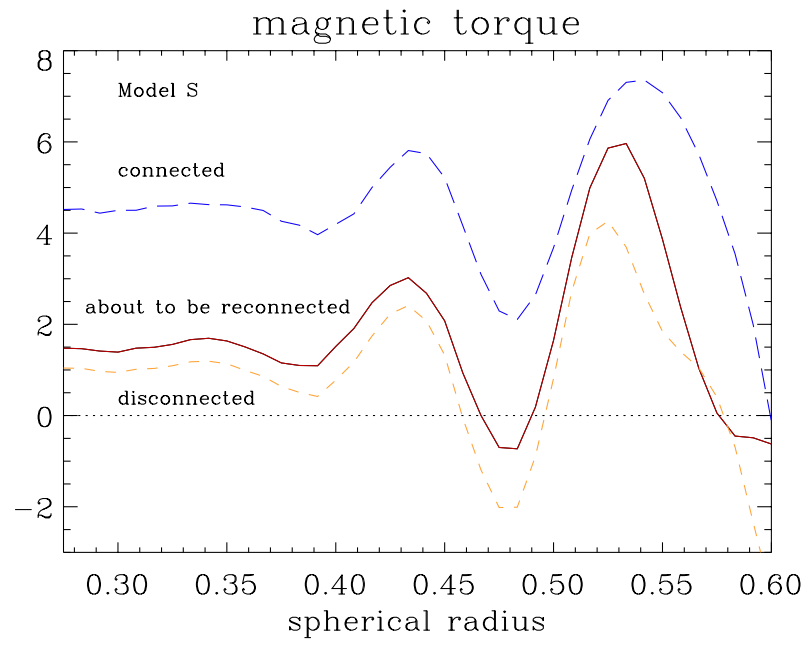

Fig. 21. Magnetic torque $T_{\operatorname{mag}}$ as function of spherical radius $r$ for Model $\mathrm{S}$ at three different times. $T_{\text {mag }}$ is shown for radii between the approximate anchoring radius $r_{0}=0.275$ and the inner disc radius $\varpi=0.6$. Blue long-dashed: $t=98$ when the star is magnetically connected to the disc; orange dashed: $t=102$ when the star is magnetically disconnected from the disc; red solid: $t=103$ when the star is about to be magnetically reconnected to the disc (cf. Fig. 20). The black dotted line marks the magnetic torqueless state $\left(T_{\text {mag }}=0\right)$.

(i.e. a material spin-down), whereas at latitudes of stellar outflow, the net accretion torque is positive (i.e. a material spinup). This is due to the small rotation rates between the star and the disc.

\section{Conclusions}

In agreement with earlier work by Hirose et al. (1997), Goodson \& Winglee (1999) and Matt et al. (2002), our work confirms the possibility of episodic and recurrent magnetospheric accretion also for models where the disc magnetic field is not imposed but dynamo-generated. The critical stellar surface field strength required for the episodic accretion to be correlated with the magnetic star-disc coupling, is around (or below) $2 \mathrm{kG}$, which is well in the range of observed field strengths for T Tauri stars (Johns-Krull et al. 1999). These recurrent changes in the connectivity between the stellar magnetosphere and the dynamo-generated disc field result in episodic mass transfer from the disc to the star. For the same stellar surface field strengths, the time-dependent wind velocities are also correlated with the magnetic star-disc coupling. The wind velocities as well as specific angular momentum transport from the disc inner edge into the corona are enhanced during periods when the star is disconnected from the disc. Highly timedependent accretion and outflows have also been detected observationally in classical T Tauri stars (CTTS) by Stempels \& Piskunov (2002, 2003).

The observed stellar fields are, however, not dipolar, but show a strong nonaxisymmetric component, although the geometry of the magnetosphere of protostars is yet unknown. (The assumption of a stellar dipolar field is motivated by observations that suggest that the stellar field might be concentrated at the poles in rapidly rotating CTTS; e.g., Schüssler et al. (1996).) The fact that the fields of T Tauri stars are not axisymmetric may have important implications for the accretion process. Our work suggests that dipolar fields tend to divert a significant fraction of disc matter into the wind. Channelling the disc material along magnetospheric stellar field lines becomes more efficient if the magnetospheric accretion process happens in an episodic fashion. It is conceivable that a nonaxisymmetric stellar field has a similar effect, but it would then produce time variability on a time scale similar to the stellar rotation period. Variability on time scales similar to the stellar orbital period has indeed been observed; see Johns-Krull et al. (1999).

We find that the sum of magnetic, accretion and viscous torques acting on the star, is positive at most radii between the star and the disc if the stellar surface field strength exceeds a certain value that is somewhere between $200 \mathrm{G}$ and $1 \mathrm{kG}$. This positive torque means a stellar spin-up, in agreement with Romanova et al. (2002) for stellar rotation periods of around 9 to 10 days. The accretion and viscous torques are almost always much smaller (in amplitude) than the magnetic torque. The small accretion torque in our simulations might be due to the relatively small accretion rates and accretion flow velocities, both of which are not imposed. These, in turn, might be due to the fixed inner disc radius.

The mean-field disc dynamo is responsible for the structure in the disc wind, with coexisting pressure-driving of the outer disc wind and magneto-centrifugal launching and acceleration of the inner disc wind. The stellar wind is always mostly pressure-driven.

In contrast to the simulations of Hirose et al. (1997) and Goodson et al. (1997), we do not find any stellar (fast) jet that is driven by magneto-centrifugal processes. However, our magneto-centrifugally driven fast inner disc wind is close to the star and might collimate at larger heights to form the observed protostellar jet (cf. Fendt et al. 1995).

Another important result that has emerged from the present investigations is an anti-alignment of the disc magnetic field relative to the stellar dipole (no X-point). In our models, the relative orientation of the disc field and the stellar field is no longer a free input parameter, but a result of the simulations. The only obvious way to prevent anti-alignment of the disc magnetic field, is to have magnetic fields in the disc and in the protostar that are entirely due to the accretion of an ambient large scale field. This may indeed be quite plausible for many protostellar discs in a large fraction of star forming regions. Another argument in favour of this possibility is the fact that strong collimation of protostellar outflows into well pronounced jets has so far only been found in the presence of a large scale field aligned with the rotation axis of the star-disc system (e.g., Ouyed et al. 1997), and not for dynamo-generated disc magnetic fields (see Paper I).

An important limitation of the present work is the restriction to axisymmetric magnetospheric accretion. Given that fully three-dimensional simulations have now become feasible (e.g., Hawley 2000), this would certainly be an important constraint to be relaxed in future simulations. 

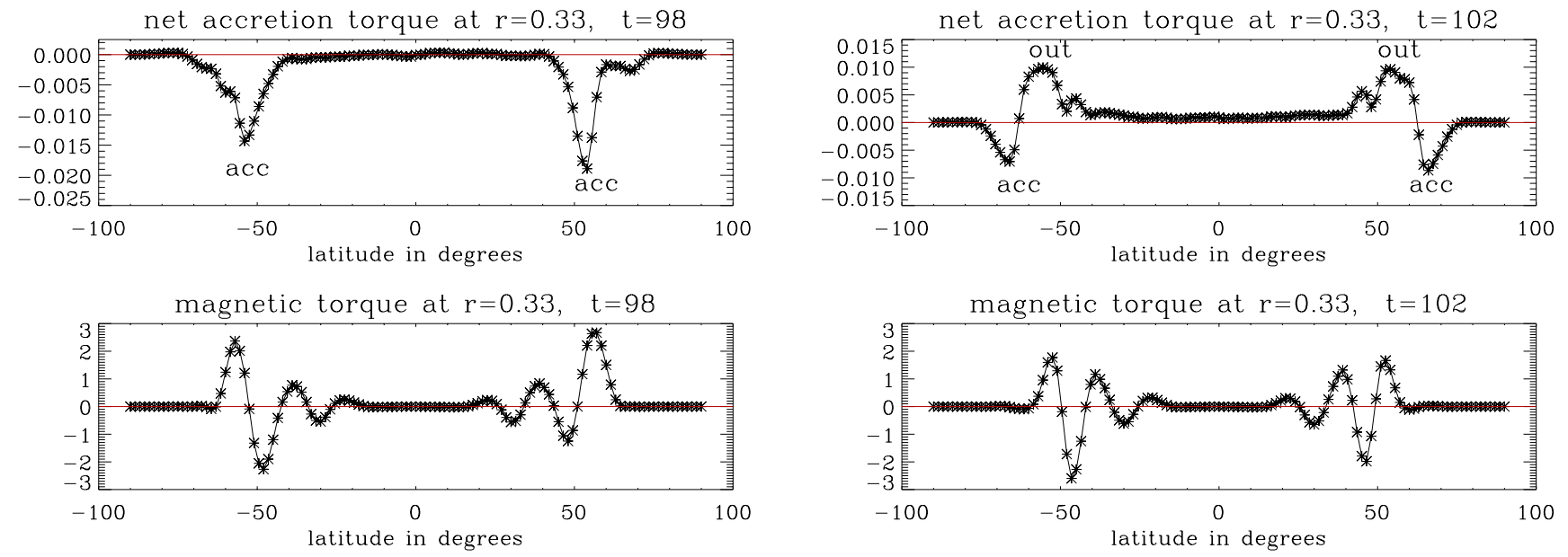

Fig. 22. Net accretion torque (upper panel) and magnetic torque (lower panel) as functions of latitude at the spherical radius $r \approx 0.33$ (close to the star) for Model S for the two distinctive states: when the star is magnetically connected to the disc $(t=98$, left $)$ and when the star is magnetically disconnected from the disc $(t=102$, right). Latitudes marked with "acc" are regions of strongest mass accretion (cf. Fig. 9) and latitudes marked with "out" are regions of strongest mass outflow (cf. Fig. 10).

Acknowledgements. Use of the supercomputer SGI 3800 in Linköping and of the PPARC supported supercomputers in St Andrews and Leicester is acknowledged. This research was conducted using the resources of High Performance Computing Center North (HPC2N). B.v.R. thanks NORDITA for hospitality. We thank Eric Blackman, Sean Matt and Ulf Torkelsson for fruitful discussions. We also thank an anonymous referee for many useful comments.

\section{Appendix A: Effective stellar rotation rate}

In this appendix we give a precise definition of the effective stellar rotation rate, $\Omega_{*}$, as it was used in Eq. (21).

Integrating Eq. (8) over the stellar volume yields an evolution equation for the total stellar angular momentum, $L_{*}$,

$\dot{L}_{*}=T_{\text {acc }}+T_{\text {mag }}+T_{\text {visc }}$,

where $L_{*}=\int \varrho l \mathrm{~d} V$ is the integral of the angular momentum density, $\varrho l$, over the stellar volume. Obviously, $L_{*}$ can grow from mass accretion alone without spinning up the star. We therefore need to look at the evolution of the specific stellar angular momentum, $l_{*}=L_{*} / M_{*}$, where $M_{*}=\int \varrho \mathrm{d} V$ is the mass of the star. Using the product rule, we can write the left hand side of Eq. (A.1) as

$\dot{L}_{*}=\dot{l}_{*} M_{*}+l_{*} \dot{M}_{*}$,

where the accretion rate $\dot{M}_{*}$ can be expressed in terms of the mass flux density, $\varrho \boldsymbol{u}_{\mathrm{pol}}$, via $\dot{M}_{*}=-\oint \varrho \boldsymbol{u}_{\mathrm{pol}} \cdot \mathrm{d} \boldsymbol{S}$, where the integral is taken over the stellar surface $(\mathrm{d} \boldsymbol{S}$ is the outward directed surface element). Further, $l_{*}=L_{*} / M_{*}=\int \varrho l \mathrm{~d} V / \int \varrho \mathrm{d} V$ can be expressed as $l_{*}=\varpi_{*}^{2} \Omega_{*}$, where $\varpi_{*}$ is defined as a weighed average,

$\varpi_{*}^{2} \equiv\left\langle\varpi^{2}\right\rangle=\oint \varrho \boldsymbol{u}_{\mathrm{pol}} \varpi^{2} \cdot \mathrm{d} \boldsymbol{S} / \oint \varrho \boldsymbol{u}_{\mathrm{pol}} \cdot \mathrm{d} \boldsymbol{S}$,

where the integrals are taken over the stellar surface. This defines $\Omega_{*}$, which replaces the intuitive definition of $\Omega_{*}$ as the stellar surface rotation rate (the latter, however, is still sufficiently accurate a definition for all practical purposes).

With this definition of $\varpi_{*}$ (and $\Omega_{*}$ ), we obtain an evolution equation for the specific stellar angular momentum, $l_{*}$,

$M_{*} \dot{l}_{*}=T_{\mathrm{acc}}-l_{*} \dot{M}_{*}+T_{\mathrm{mag}}+T_{\mathrm{visc}}$,

which can be written as

$M_{*} \dot{l}_{*}=T_{\text {acc,net }}+T_{\text {mag }}+T_{\text {visc }}$

with $T_{\text {acc,net }}$ given in Eq. (21). This shows that the sign of the right hand side of Eq. (A.5) determines whether the star spins up or down.

\section{References}

Agapitou, V., \& Papaloizou, J. C. B. 2000, MNRAS, 317, 273

Bardou, A. 1999, MNRAS, 306, 669

Bardou, A., von Rekowski, B., Dobler, W., et al. 2001, A\&A, 370, 635

Blandford, R. D., \& Payne, D. R. 1982, MNRAS, 199, 883

Brandenburg, A. 1998, in Theory of Black Hole Accretion Discs, ed. M. A. Abramowicz, G. Björnsson, \& J. E. Pringle (Cambridge University Press), 61

Brandenburg, A., Nordlund, ̊., Stein, R. F., et al. 1995, ApJ, 446, 741

Brandenburg, A., Tuominen, I., \& Krause, F. 1990, GAFD, 50, 95

Cameron, A. C., \& Campbell, C. G. 1993, A\&A, 274, 309

Campbell, C. G. 1999, MNRAS, 310, 1175

Campbell, C. G. 2000, MNRAS, 317, 501

Campbell, C. G. 2001, MNRAS, 323, 211

Dobler, W., Shukurov, A., \& Brandenburg, A. 2002, Phys. Rev. E, 65, 036311

Fendt, C., Camenzind, M., \& Appl, S. 1995, A\&A, 300, 791

Ghosh, P., \& Lamb, F. K. 1979a, ApJ, 232, 259

Ghosh, P., \& Lamb, F. K. 1979b, ApJ, 234, 296

Ghosh, P., Lamb, F. K., \& Pethick, C. J. 1977, ApJ, 217, 578

Goodson, A. P., Böhm, K.-H., \& Winglee, R. M. 1999, ApJ, 524, 142

Goodson, A. P., \& Winglee, R. M. 1999, ApJ, 524, 159

Goodson, A. P., Winglee, R. M., \& Böhm, K.-H. 1997, ApJ, 489, 199

Guenther, E. W., Lehmann, H., Emerson, J. P., et al. 1999, A\&A, 341, 768 
Janhunen, P., \& Huuskonen, A. 1993, JGR, 98, 9519

Johns-Krull, C. M., \& Valenti, J. A. 2001, ApJ, 561, 1060

Johns-Krull, C. M., Valenti, J. A., \& Koresko, C. 1999, ApJ, 516, 900

Hawley, J. F. 2000, ApJ, 528, 462

Hawley, J. F., \& Balbus, S. A. 1991, ApJ, 376, 223

Hayashi, M. R., Shibata, K., \& Matsumoto, R. 1996, ApJ, 468, L37

Hirose, S., Uchida, Y., Shibata, K., et al. 1997, Publ. Astron. Soc. Japan, 49, 193

Königl, A. 1991, ApJ, 370, L39

Krause, F., \& Rädler, K.-H. 1980, Mean-Field Magnetohydrodynamics and Dynamo Theory (Berlin: Akademie-Verlag)

Küker, M., Henning, T., \& Rüdiger, G. 2003, ApJ, 589, 397

Lovelace, R. V. E., Romanova, M. M., \& Bisnovatyi-Kogan, G. S. 1995, ApJ, 275, 244

Matt, S., Goodson, A. P., Winglee, R. M., et al. 2002, ApJ, 574, 232

Miller, K. A., \& Stone, J. M. 1997, ApJ, 489, 890

Ouyed, R., Pudritz, R. E., \& Stone, J. M. 1997, Nature, 385, 409

Papaloizou, J. C. B., \& Terquem, C. 1999, ApJ, 521, 823
Parker, E. N. 1979, Cosmical Magnetic Fields (Oxford: Clarendon Press)

Pelletier, G., \& Pudritz, R. E. 1992, ApJ, 394, 117

von Rekowski, B., Brandenburg, A., Dobler, W., et al. 2003, A\&A, 398, 825 (Paper I)

von Rekowski, M., Rüdiger, G., \& Elstner, D. 2000, A\&A, 353, 813

Reyes-Ruiz, M., \& Stepinski, T. F. 1995, ApJ, 438, 750

Romanova, M. M., Ustyugova, G. V., Koldoba, A. V., et al. 2002, ApJ, 578,420

Schüssler, M., Caligari, P., Ferriz-Mas, A., et al. 1996, A\&A, 314, 503

Shu, F., Najita, J., Ostriker, E., et al. 1994, ApJ, 429, 781

Stempels, H. C., \& Piskunov, N. 2002, A\&A, 391, 595

Stempels, H. C., \& Piskunov, N. 2003, A\&A, 408, 693

Tuominen, I., Brandenburg, A., Moss, D., et al. 1994, A\&A, 284, 259

Urpin, V., \& Brandenburg, A. 1998, MNRAS, 294, 399

Yousef, T. A., Brandenburg, A., \& Rüdiger, G. 2003, A\&A, 411, 321

Ziegler, U., \& Rüdiger, G. 2000, A\&A, 356, 1141 